Vargas Girón, J.M. (2020): “Ofrendas de carácter pesquero en santuarios litorales: el caso de La Algaida (Sanlúcar de Barrameda, Cádiz)", Spal 29.2: 235-257. DOI: https://dx.doi.org/10.12795/spal.2020.i29.25

\title{
OFRENDAS DE CARÁCTER PESQUERO EN SANTUARIOS LITORALES: EL CASO DE LA ALGAIDA (SANLÚCAR DE BARRAMEDA, CÁDIZ)
}

\author{
OFFERINGS OF FISHERY NATURE IN COASTAL SANCTUARIES: \\ THE CASE OF LA ALGAIDA (SANLUCAR DE BARRAMEDA, CADIZ)
}

\author{
JOSÉ MANUEL VARGAS GIRÓN \\ Profesor Sustituto Interino \\ Departamento de Historia, Geografía y Filosofía. Área de Arqueología. \\ Facultad de Filosofía y Letras. Universidad de Cádiz \\ Avda. Dr Gómez Ulla s/n 11003, Cádiz \\ Correo-e: josemanuel.vargas@uca.es (D https://orcid.org/0000-0003-2882-8902 \\ WoS ResearcherID: <https://publons.com/researcher/AAU-6452-2020>
}

Resumen: Las excavaciones arqueológicas realizadas en el santuario fenicio-púnico de La Algaida (Sanlúcar de Barrameda) durante los años 70 y 80 del pasado siglo han permitido recuperar más de diez mil exvotos de muy diversa naturaleza, tanto de producción local como procedentes de diferentes puntos del Mediterráneo, los cuales abarcan un espectro cronológico muy amplio comprendido entre los siglos VII-I a.C. A través del análisis de estos materiales se han podido reconstruir aspectos religiosos de gran interés como pueden ser las prácticas rituales que se celebraban en este espacio sagrado, así como determinar cuáles fueron las divinidades allí veneradas. En este trabajo se dan a conocer por primera vez los instrumentos de pesca procedentes de este yacimiento, presentándose, en primer lugar, un análisis morfo-tipológico de los mismos y, en segundo lugar, una valoración de este tipo de material arqueológico en contextos sagrados, planteándose los diferentes usos y funciones que pudieron haber tenido las artes y aparejos de pesca en los santuarios antiguos.

Palabras claves: Exvotos, artes de pesca, La Algaida, época fenicio-púnica.

\begin{abstract}
The archeological digs carried out in the Phoenician-Punic sanctuary of La Algaida (Sanlucar de Barrameda) during the 70 s and 80 s decade of the last century have allowed the recovery of more than ten thousand votive offerings from many different natures, from local-produced ones to others coming from different points in the Mediterranean Sea. They span a wide chronological spectrum covering from 7th to 1st Centuries BC. Thanks to the analysis of these materials it has been possible to reconstruct religious aspects of great interest. Aspects such as the ritual practices that were held in this sacred space or the possibility to determine who were the deities that were worshiped there. The fishing tools coming from this archeological site are presented in this work for the first time and are presented first in a morpho-typological analysis of the materials, and secondly, in an evaluation of this kind of archeological material in sacred contexts, considering the different uses and functions that the fishing gears and skills could have had in the ancient places of worship.

Keywords: Votive offerings, fishing gear, La Algaida, phoenician-punic times.
\end{abstract}




\section{EL SANTUARIO FENICIO-PÚNICO DE LA ALGAIDA. CONTEXTUALIZACIÓN HISTÓRICA Y PROBLEMÁTICA ARQUEOLÓGICA}

A lo largo de nuestra investigación se han inventariado un total de novecientas quince evidencias de instrumental de pesca (Vargas 2017a), las cuales proceden de tres áreas geográficas cuya estrecha relación con actividades pesqueras está bien documentada a nivel arqueológico desde época muy antigua: Cádiz, Murcia y Alicante (Vargas 2017b: 132). Por lo que respecta al Fretum Gaditanum, una de las zonas que mayor cantidad de información nos ha proporcionado ha sido la fachada atlántica gaditana, donde tenemos que destacar dos enclaves marítimos muy importantes como son Sanlúcar de Barrameda y Chipiona que, por su estratégica situación en la desembocadura del río Guadalquivir, han constituido lugares de obligado paso a lo largo de la Antigüedad. De hecho, uno de los yacimientos estudiados donde han aparecido un mayor número de piezas ha sido el santuario de La Algaida -Sanlúcar de Barrameda, Cádiz- (fig. 1), habiéndose recuperado un total de cuarenta y dos instrumentos de pesca. De todos los sitios arqueológicos estudiados (sesenta en total), La Algaida constituye el único yacimiento relacionado con actividades de culto, de ahí la importancia de los materiales arqueológicos que se presentan en este trabajo.

El yacimiento de La Algaida, también conocido como Monte Algaida o Cerro del Tesorillo (Ferrer 2002a: 107), está situado en el pinar de la Algaida, en la marisma de Bonanza, al norte de la localidad de Sanlúcar de Barrameda. Desde un punto de vista paleotopográfico, estaría emplazado en una isla alargada de formación aluvial, cuyo eje más largo se dispondría en dirección suroeste-noreste y presentaría cuatro kilómetros y medio de longitud, mientras que su anchura alcanzaría un kilómetro y medio (Corzo 2007: 195). Si atendemos a la reconstrucción paleotopográfica de la desembocadura del río Guadalquivir (Ménanteau 1982), la península o lengua arenosa donde se ubicaría el santuario de La Algaida es el resultado de la unión entre la orilla oriental del río y lo que fue, durante el I milenio antes de nuestra era, la orilla occidental del antiguo cauce (Corzo 2007: 195).

Las primeras noticias que tenemos del santuario de La Algaida se la debemos al estudioso local Pedro Barbadillo (1951), quien realizó varias prospecciones durante los años 40 del pasado siglo en la zona del bosque más cercana a las marismas inferiores del Guadalquivir (zona B), deteniéndose especialmente en una duna conocida como Cerro del Tesorillo (Pérez López 1998), que llegó a identificar con Tartesos (Ferrer 2002b: 198). Unos años más tarde se iniciaría la construcción de un camino forestal para facilitar el aprovechamiento de las maderas del lugar, descubriéndose unas "vetas de piedra", a lo que tenemos que sumar la aparición de materiales de época romana (Esteve 1952: 126), motivo por el cual el alcalde de la localidad encargó una campaña de excavación a Esteve Guerrero cuyos resultados preliminares publicaría en el año 1952. Tuvieron que pasar nada más y nada menos que casi treinta años para que se reanudaran las investigaciones arqueológicas en este yacimiento, gracias a la llegada de un equipo del Museo de Cádiz liderado por Ramón Corzo - director del museo por aquellas fechas-, sucediéndose seis campañas de excavación de manera sistemática entre 1978 y 1984.

Durante estos siete años de excavaciones se descubrieron las tres cuartas partes del yacimiento. La fase fenicio-púnica del área excavada corresponde a un temenos o espacio abierto sagrado, donde supuestamente se situaría el betilo o la piedra de altar para los sacrificios (Corzo 1984: 144) -que no se ha conservado-. Lo que sí se ha documentado ha sido un pozo lustral alrededor del cual se construyeron tres pequeños edificios que, según Ramón Corzo (1984: 144), podrían responder a épocas y técnicas distintas (fig. 2 a). En cualquier caso, los tres presentaban concentraciones de objetos en sus rincones. El edificio de mayores dimensiones es, a su vez, el mejor conservado, presentando una compartimentación interna de dos habitaciones, al más puro estilo griego (pronaos y cella). Otro de los edificios, que parece ser el más antiguo, se caracteriza por estar construido a base de muros muy gruesos de mampostería, generándose un "tesoro" de 3 metros de lado. Su antigüedad viene determinada porque en su interior han aparecido piezas de bronce de origen etrusco (Corzo 1991). Muy cerca de estos thesauri, aparece un edificio alargado compartimentado en tres habitaciones, junto al que aparecieron cenizas, huesos de animales y una mayor cantidad de fragmentos anfóricos con respecto al resto del yacimiento, razón por la cual ha sido interpretado como la casa de los oficiales del santuario (Corzo 1984: 144). El resto de las ofrendas se depositaron a lo largo del temenos, lo que originó la acumulación y remoción de objetos desde la Antigüedad, imposibilitando, de este modo, distinguir fases de uso (Ferrer 2002a: 108). Desde esta perspectiva, la estratigrafía del yacimiento presenta una lectura confusa debido principalmente a la propia formación del estrato: 


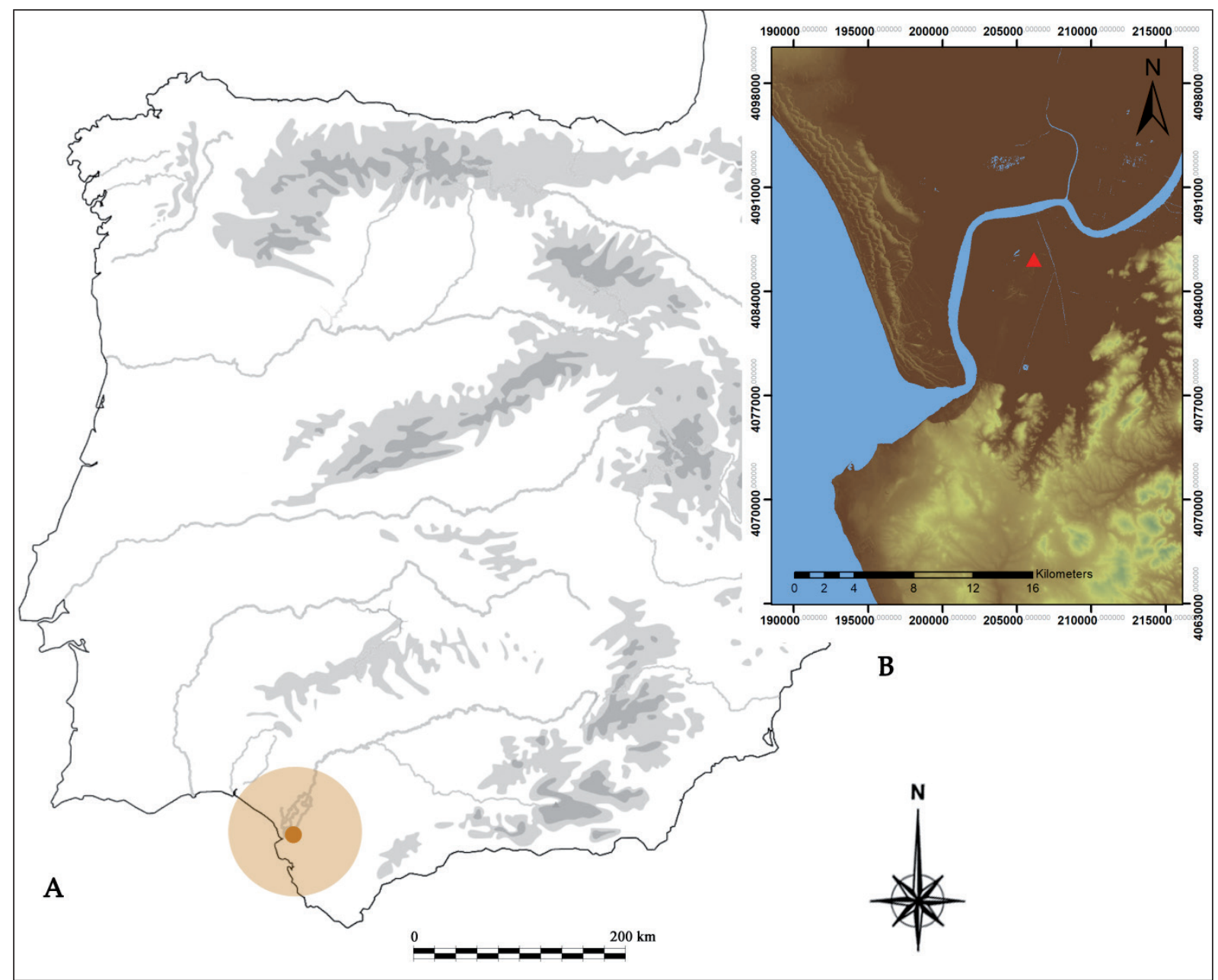

Figura 1. Localización geográfica del santuario de La Algaida en el suroeste de la Península Ibérica (A) y en las proximidades de la desembocadura del río Guadalquivir (B).

posiblemente se produjo una acumulación de ofrendas procediéndose a su retirada en un momento dado, de ahí que se hayan recogido objetos de cronologías tan dispares (Ferrer 2002b: 199). Generalmente, los materiales de los siglos VII-VI a.C. son muy escasos, mientras que el grueso corresponde a los siglos IV-II a.C.

El yacimiento se situó sobre una duna de aluvión natural de escasa altura, conformándose como el propio pavimento del complejo sacro. Sobre este nivel dunar se depositó una gran variedad de ofrendas que, mezcladas con cenizas y materia orgánica, conformaron un estrato de potencia variable pero no superior a los 80 $\mathrm{cm}$, que estaba asociado a las construcciones prerromanas. Las cellae están amortizadas por niveles de derrumbe compuestos por acumulaciones de arena (Ferrer 2002b: 199). Sobre estos niveles se deposita un estrato irregular de época romana con materiales julio-claudios - correspondientes a una supuesta factoría de salazones (fig. 2 b) - y tardorromanos - pertenecientes a estructuras funerarias- (Esteve 1952).

La cuestión estratigráfica ha sido uno de los principales problemas a la hora de establecer los límites cronológicos del santuario. Sin embargo, algunos materiales de cronología arcaica sugieren una sacralización del espacio al menos desde los siglos VII-VI a.C., prolongándose hasta los siglos II-I a.C. En este sentido, los materiales de mayor antigüedad se corresponden con una garra felina de bronce perteneciente a una de las tres patas de un timiaterio o algún otro soporte ritual fenicio del siglo VI a.C., así como a una fíbula tipo Alcores -siglo VII a.C.- (Ferrer 2002b: 199). Por su parte, las últimas ofrendas podrían pertenecer, por un lado, a 
vasos de producción campaniense y, por otro lado, a ánforas púnicas Maña $\mathrm{C} 2 \mathrm{a}$, fechables en la primera mitad del siglo I a.C., a lo que tenemos que sumar otros indicadores cronológicos tales como la cita de Estrabón (3.1.9) y la construcción de una posible cetaria de época romana, que marcaría el final de las actividades de culto en La Algaida (Ferrer 2002a: 109). Otros autores prefieren acortar el periodo de vida del santuario sanluqueño, estableciéndose una cronología que abarca desde el siglo VI al II a.C. (Corzo 1984: 143). Los hallazgos monetales, sin embargo, plantean un funcionamiento del santuario entre los siglos IV-I a.C., aunque es posible que siguiera siendo visitado durante el siglo I d.C., perdiendo su importancia a partir del siglo II d.C. y volviendo a tener interés en la segunda mitad del siglo IV d.C. (López de la Orden y Blanco 2000: 491). En cualquier caso, parece ser que el auge del santuario tuvo lugar durante los siglos IV-III a.C., a tenor de la cronología de las cerámicas, que constituyen los objetos más frecuentemente representados (Ferrer 2014: 226).

Las memorias de las excavaciones realizadas por Corzo en el santuario de La Algaida permanecen inéditas, por lo que el análisis del instrumental pesquero que traemos a colación se ha visto parcialmente sesgado. Sin embargo, tenemos ciertos datos acerca de la estratigrafía del yacimiento que pueden ser tomados para el estudio de nuestras piezas. De manera genérica se distinguen cinco niveles (Blanco Frejeiro y Corzo 1983):

1) Un primer nivel edáfico correspondiente a la época de la excavación (nivel I).

2) Por debajo se encontraría el nivel II, que constituye un estrato de arenas donde aparecen restos discontinuos de época julio-claudia que pueden tener bastante relación con los niveles excavados por Esteve (1952) en los años 40 y que él mismo puso en relación con una factoría de salazón romana, interpretación que se ha puesto en duda en investigaciones posteriores. Para Ramón Corzo (2007: 197), las estructuras arquitectónicas descubiertas por Esteve podrían corresponder a una casa y a un taller de un carpintero de ribera de época romana altoimperial. Para este autor la ausencia de piletas y otras evidencias relacionadas con la industria pesquero-conservera es la principal razón para descartar la interpretación de la factoría de salazones. Por otro lado, en la Red de Excelencia Atlántico-Mediterránea del Patrimonio Pesquero de la Antigüedad (RAMPPA: http://ramppa.uca.es/) no se ha incluido una ficha específica de La Algaida ya que los responsables de dicho laboratorio virtual consideran que no hay argumentos de peso para confirmar que estas estructuras pertenezcan a una cetaria. Además de estos restos, se documentan también tumbas destruidas con una cronología que abarca desde el siglo II al siglo IV d.C.

3) Nivel de arenas eólicas (nivel III) que marcan el momento final del santuario. Estas arenas, las cuales presentan un grosor variable si bien en algunos puntos llegan a alcanzar un metro de potencia, aparecen amortizando las construcciones del santuario.

4) En el nivel IV es donde aparece el santuario fenicio-púnico. Se caracteriza por la documentación de estructuras formadas por cimientos de guijarros y muros de adobe (Corzo 1984: 144), así como por la localización de abundante material arqueológico.

5) Por último se documenta la paleoduna sobre la que se asentó el yacimiento (nivel V). Esta base constituye el terreno de aluvión natural del pinar, situándose a poco más de un metro de altura sobre el nivel freático del río (Corzo 1991: 401).

De todos los niveles que se han descrito nos interesa fundamentalmente el nivel IV, pues es aquí donde aparece la gran mayoría del material mueble, representado principalmente por cerámicas y metales. El sedimento de este nivel se caracteriza por presentar una matriz arenosa de coloración oscura, que es el resultado de la descomposición de la materia orgánica de las ofrendas líquidas, de ahí la abundante aparición de vajilla cerámica de mesa. Junto al material cerámico también aparecieron restos de peces, que debieron ser servidos en los conocidos platos de pescado, de los cuales se han recogido numerosos fragmentos. De la misma manera, merecen ser destacados igualmente los donativos de terracotas femeninas, elementos de joyería y metales preciosos (López Amador y Ruiz Gil 2010: 273).

Otra de las cuestiones que ha generado una gran controversia entre los investigadores ha girado en torno a la determinación de la divinidad adorada en este enclave sagrado. Para Ferrer (2002b) esta problemática tendría que ser analizada desde tres perspectivas diferentes: geográfica/toponímica, iconográfica y mitológica, siendo la primera de ellas la que ha generado opiniones más unánimes. En este sentido, el santuario de La Algaida, cuyo carácter costero queda patente por su situación geográfica, se localiza en la desembocadura del río Guadalquivir, ocupando un lugar estratégico para los navegantes que circundaban las aguas del antiguo Lacus Ligustinus, bien para adentrarse hacia el río Baítis bien para salir hacia las aguas del Atlántico. Su carácter cosmopolita se ha podido determinar, 


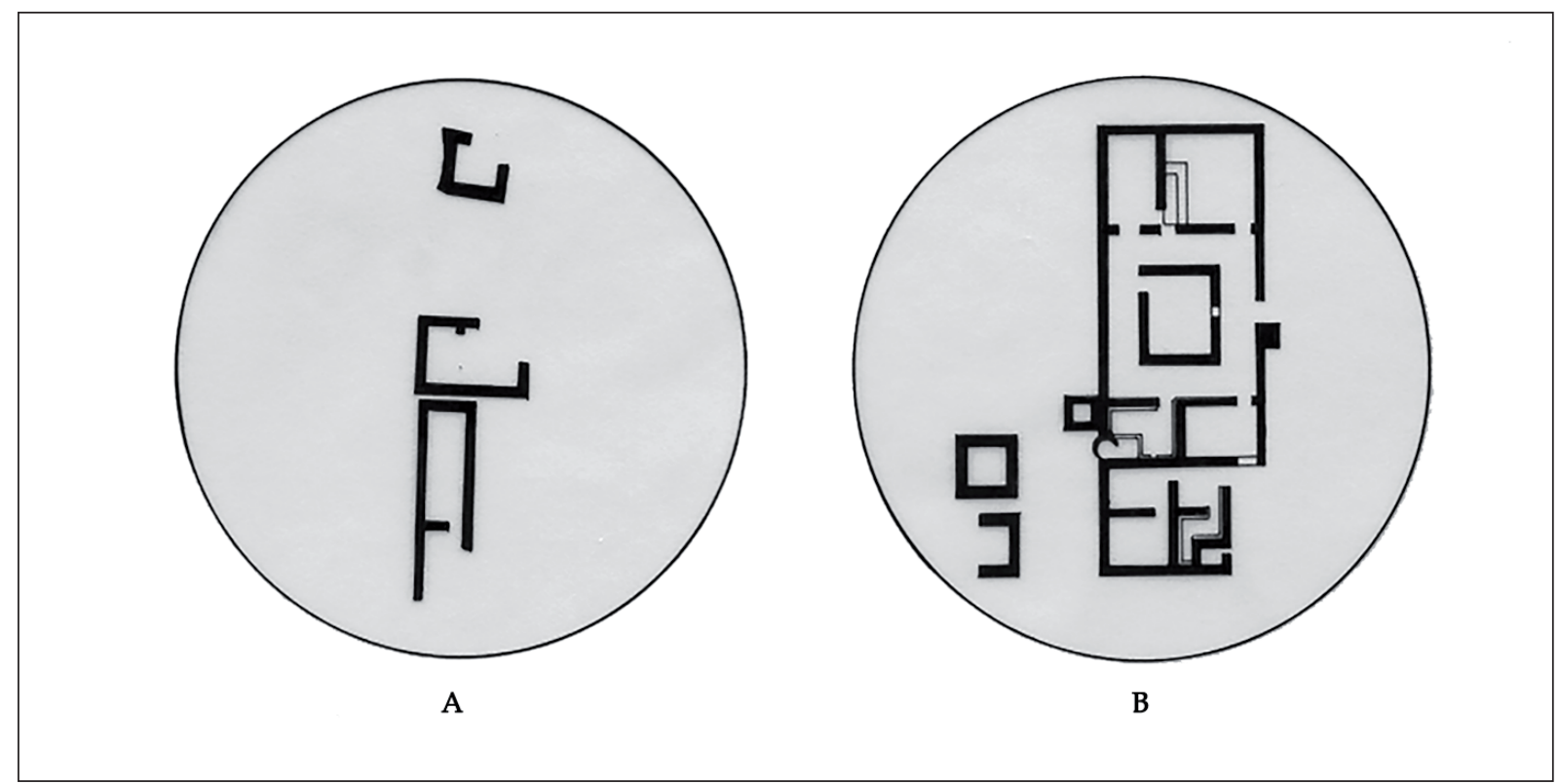

Figura 2. Planta de las estructuras arquitectónicas correspondientes al santuario fenicio-púnico (A) y a la supuesta factoría de salazones de época romana excavada por Esteve (B) (a partir de Pérez López 1998).

además, gracias a la aparición de materiales procedentes de diferentes puntos del Mediterráneo -Grecia, Etruria y Egipto- y con una notable variedad de influencias artísticas (Corzo 2007: 200), a lo que tenemos que sumar toda una amplia gama de productos locales de fabricación turdetana (Corzo 1991: 403). Desde esta perspectiva, la identificación del santuario de La Algaida con la diosa Fósforo (Phosphoros) o "Luz Incierta" (Lux Dubia) mencionado por Estrabón en el tercer libro de su Geografía (3.1.9), permite plantear su relación con una diosa euploia, es decir, protectora de la navegación y guía de los navegantes (Ferrer 2002a: 109). La cita dice así: «Partiendo de allí encontramos la corriente del Betis, la ciudad de Ébura y el santuario de la diosa Fósforo, a la que llaman Luz Incierta» (trad. Meana y Piñero 1992).

Esta Lux Dubia o luz dudosa se ha relacionado con la luz del crepúsculo, esto es, el lucero del anochecer, siendo uno de los astros más llamativos de nuestro cielo, que se asocia desde muy antiguo con la diosa Venus (Corzo 1991: 400).

Sin embargo, es el análisis de las ofrendas el que puede aportarnos una mayor cantidad de información en este sentido, habiéndose podido determinar que las advocaciones y los poderes de la diosa allí venerada eran mucho más amplios (Ferrer 2002b: 202):

- En primer lugar, y en relación con la cita de Estrabón, tenemos que destacar la frecuente entrega de lámparas de aceite encendidas a modo de lucernas, las cuales redundan en el carácter luminoso de la divinidad (Ferrer 2002b: 202).

- El carácter marinero del santuario queda también demostrado por las ofrendas de conchas y peces (Ferrer 2002b: 202), a lo que tenemos que sumar la singular aparición de instrumentos de pesca, que constituyen los materiales objeto de este trabajo. Pérez López (1998) incide en la naturaleza marítima de algunos materiales del santuario a la hora de identificar a la divinidad tutelar de este recinto, mencionando la aparición de vértebras de pescado, conchas de moluscos, dientes de tiburón, anzuelos y resaltando la temática marina de algunos anillos.

- No menos interés tienen los pebeteros o cabezas de terracota utilizadas como timiaterio así como las joyas con representaciones de temas animales y vegetales, lo que induce a pensar en el poder ejercido por la diosa sobre la naturaleza (Ferrer 2002b: 202). La presencia de timiaterios en forma de cabeza femenina en el santuario de La Algaida se ha puesto en relación con un ritual consistente en quemar incienso y perfumes durante las ceremonias del culto (Pérez López 1998, Corzo 2007: 207).

- Los amuletos de tipo apotropaico están claramente vinculados a los poderes curativos de la divinidad (Ferrer 2002b: 202). Dentro de esta categoría, destacan principalmente las placas de plata con la 
representación de un par de ojos que, además de incidir en el carácter salutífero de la deidad allí venerada, podrían estar conectadas con el culto a una divinidad cuyos poderes se relacionan con la luz (Pérez López 1998).

- De gran interés son las figuras de terracota de carácter curótrofo representando a mujeres embarazadas y con niños en brazos, que han permitido vincular el santuario con una diosa de la fertilidad con una clara advocación maternal y nutricia (Ferrer 2002a: 109, 2002b: 202).

Por lo tanto, cabría preguntarnos cuál sería la divinidad que albergaría todas estas advocaciones. Para Eduardo Ferrer, la sacralización de la isla de La Algaida se habría producido en época fenicia arcaica bajo la advocación de Astarté, siendo una divinidad marinera en tanto en cuanto guiaba y protegía a los navegantes. Sin embargo, cuando se produjo la monumentalización del santuario como tal (desde el siglo IV a.C. o un poco más tarde) pudo haberse producido una sustitución o un sincretismo con Tinnit, gracias a una mayor influencia de Cartago (Ferrer 2002b: 202).

Hasta la fecha se han dado a conocer diferentes tipos de objetos votivos $\mathrm{u}$ ofrendas procedentes del santuario. Es el caso de las monedas (López de la Orden y Blanco 2000), para las cuales se ha planteado una doble función: en primer lugar, se ha defendido que el principal uso de la moneda en el santuario de La Algaida es de carácter votivo; en segundo lugar, tampoco se descarta su uso monetal, sobre todo para comprar las ofrendas que luego se ofrecerían a la divinidad. También se han publicado fíbulas (Storch de Gracia 1989), cuentas de collar de pasta vítrea, cornalina y conchas (Ruano et al. 1996), escarabeos y amuletos (López de la Orden 1990), bronces (Corzo 1991), anillos (Corzo 2000: 154-164, López Amador y Ruiz Gil 2010) y terracotas (Corzo 2007).

El objetivo de este trabajo es dar a conocer una serie de materiales inéditos de carácter pesquero sobre los cuales apenas se ha llamado la atención hasta el momento, valorándose su más que posible atribución votiva. Algunos autores, sin embargo, han mencionado la aparición de artes de pesca en La Algaida, fundamentalmente anzuelos de bronce (Pérez López 1998, Ferrer 2002a: 112, López Amador y Ruiz Gil 2010: 273), entre las ofrendas del santuario. Ángel Muñoz ha puesto en relación, recientemente (Muñoz 2012: 63), el instrumental pesquero de La Algaida con las ofrendas halladas en el santuario, algo que no debería de extrañarnos si tenemos en cuenta que nos encontramos ante un santuario costero con una clara advocación marinera. A pesar de todo, poco o casi nada se sabe acerca de los instrumentos de pesca depositados en este enclave sagrado.

Los materiales que aquí se presentan pertenecen a los fondos del Museo de Cádiz y han sido estudiados en el marco del Proyecto de Investigación de Excelencia SAGENA -HUM-03015- (Bernal 2009, BekkerNielsen y Bernal 2010, Bernal 2011), cuyo principal objetivo no ha sido otro que documentar arqueológicamente las diferentes artes de pesca tradicionalmente utilizadas en el sur de la península ibérica. Generalmente, se desconocen datos arqueológicos precisos de los contextos donde se produjeron los hallazgos de cada una de las piezas, si bien en las fichas de inventario de las mismas hemos encontrado información que puede resultar de vital interés en este sentido.

En el caso de los anzuelos, han sido inventariados con una doble numeración: 27655 y 28025 . Con el $n^{\circ} 27655$ encontramos dos ejemplares (n. ${ }^{\text {os }} 1$ y 2 ) de los cuales tan solo sabemos que proceden de las excavaciones realizadas por Ramón Corzo en La Algaida. Por el contrario, con el $\mathrm{n}^{\mathrm{o}} 28025$ se han inventariado trece anzuelos (n. $\left.{ }^{\text {os }} 3-15\right)$ en cuya ficha de registro se especifica el año de aparición (campaña de 1981), indicándose que las piezas pertenecen a la fase fenicio-púnica del yacimiento.

Por lo que respecta a las pesas de plomo, han sido catalogadas con una triple numeración: 27679, 27674 y 28064. Los n. ${ }^{\text {os }} 27679$ (n. ${ }^{\text {os }} 2,5,6,11$ y 12) y 27674 (n. ${ }^{\text {os }}$ 1 y 3 ) corresponden a un "lote de fragmentos de plomo de diferentes formas", los cuales proceden de las excavaciones dirigidas por Ramón Corzo en el yacimiento. Por su parte, el $n^{\circ} 28064$ constituye un número de inventario que engloba una serie de piezas (n. ${ }^{\text {os }} 4,7-10$ y 13) recuperadas durante la campaña de 1983 y proceden de los niveles 3 y 4, es decir, de la fase de amortización de las estructuras del santuario, así como del período de funcionamiento del mismo.

En lo que se refiere a las agujas, se les han asignado diferentes números de inventario -27668 ( $\mathrm{n}^{\circ}$ 5), 27584 (n. ${ }^{\text {os }} 1$ y 3 ) y $27689\left(n^{\circ} 4\right)$, si bien en la ficha de registro no aparecen datos del contexto arqueológico de los materiales a excepción de la pieza $n^{\circ} 6\left(n^{\circ}\right.$ de inventario 28073), que sabemos que fue recuperada en la campaña de 1981. Una de las agujas incluidas en este estudio carece de $\mathrm{n}^{\mathrm{o}}$ de inventario, encontrándose actualmente expuesta en la denominada sala "Cádiz y la colonización fenicia" del citado museo y más concretamente dentro de la vitrina destinada al santuario de La Algaida $\left(n^{\circ} 2\right)$. 
Por último, las lanzaderas han sido igualmente inventariadas con diferente numeración: $27421\left(\mathrm{n}^{\mathrm{o}} 1\right)$, 27422 (n. ${ }^{\text {ss }} 2$ y 3), $27667\left(n^{\circ} 4\right), 27581\left(n^{\circ} 5\right)$ y 17263 $\left(n^{\circ} 6\right)$. Aquellos ejemplares inventariados con los $n .^{\text {os }}$ 27421 y 27422 fueron recuperados en la campaña de 1983 y proceden del nivel 3 (cuadro "GE”), mientras que del resto de las lanzaderas la información de la que disponemos es prácticamente nula.

\section{ESTUDIO MORFO-TIPOLÓGICO DEL INSTRUMENTAL PESQUERO}

El número total de piezas estudiadas asciende a la cifra de cuarenta y una, entre las cuales se encuentran anzuelos, pesas de plomo, agujas de bronce y de hueso y lanzaderas. Un alto porcentaje de los materiales corresponden a anzuelos simples (37\%) -que constituye la categoría de instrumental pesquero que aparece con mayor frecuencia en el registro arqueológico- $\mathrm{y}$ a pesas fabricadas con láminas de plomo (34\%), que constituyen un modelo de lastre ampliamente difundido en ámbito atlántico-mediterráneo desde el Bronce Final Egeo (Pulak 1988), tal y como han demostrado los hallazgos del pecio de Ulu Burun (Kas, Turquía). Le siguen las lanzaderas (15\%), las agujas de bronce $(12 \%)$ y las agujas de hueso $(2 \%)$.

\subsection{Anzuelos}

Del santuario de La Algaida proceden un total de quince anzuelos de bronce (n. $\left.{ }^{\text {os }} 1-15\right)$ que responden en todos los casos a modelos simples (fig. 3), que son aquellos que se caracterizan por presentar un vástago más o menos rectilíneo y una curvatura rematada en punta que normalmente suele albergar un arpón. La denominación de este tipo de anzuelos -"simple"- viene determinada por su similitud a los ganchos tradicionales utilizados en la pesca actual, con los cuales comparten muchas analogías tanto desde un punto de vista morfo-tipológico como en clave tecnológica.

Uno de los criterios que se ha planteado como potencialmente más operativo para la clasificación de los anzuelos antiguos es el relativo al tamaño. Para nuestro estudio hemos utilizado la clasificación elaborada por Bernal (2010: 89-90) para los ejemplares del Círculo del Estrecho, basada en la longitud o altura total de las piezas. De tal manera dispondríamos de ejemplares muy pequeños (menos de 2,5 cm de longitud total), pequeños (entre 2,5 y $4 \mathrm{~cm})$, medianos $(4-8 \mathrm{~cm})$ y los de tamaño excepcional o grandes, que son aquellos que superan los $8 \mathrm{~cm}$ de longitud.

Por lo que respecta a sus dimensiones (tab. 1), el primer dato importante que tenemos que destacar es la variedad de tamaños, habiéndose documentado ejemplares de ínfimas dimensiones -como es el caso del anzuelo $\mathrm{n}^{\mathrm{o}} 15$, que presenta tan solo 1,2 cm de altura- así como otros de mayor altura, tal y como observamos en el ejemplar $n^{\circ} 4$, cuyo vástago alcanza $4,8 \mathrm{~cm}$ de longitud. En cualquier caso, la gran mayoría de las piezas responden a modelos muy pequeños (n. $\left.{ }^{o s} 1,2,7-15\right)$, registrándose once ejemplares con alturas comprendidas entre 1,2 y $2,4 \mathrm{~cm}$. Por otra parte, los anzuelos de pequeño y mediano tamaño están escasamente representados -con tan solo dos ejemplares en cada caso (n. ${ }^{\text {os }} 5$ y 6 y n. ${ }^{\text {os }} 3$ y 4 respectivamente)-, a lo que tenemos que sumar la total ausencia de ganchos que superen los 8 $\mathrm{cm}$ de altura (grandes).

La anchura parece ser más uniforme que la altura. En este sentido, los anzuelos de ínfimo tamaño o muy pequeños presentan medidas oscilantes entre $1\left(n^{\circ} 14\right) y$ $1,8 \mathrm{~cm}\left(\mathrm{n}^{\circ} 7\right)$, mientras que la anchura de los ejemplares pequeños está comprendida entre $1,6\left(\mathrm{n}^{\circ} 5\right)$ y $2,5 \mathrm{~cm}\left(\mathrm{n}^{\circ}\right.$ $6)$. Por su parte, las piezas de mediano tamaño alcanzan medidas entre $1,4\left(n^{\circ} 4\right)$ y $1,7 \mathrm{~cm}$ de anchura $\left(\mathrm{n}^{\circ} 3\right)$. Por lo tanto, se observa que no existe correspondencia métrica entre los anzuelos de mayor/menor altura y aquellos de mayor/menor anchura.

Otro de los aspectos que puede derivarse del análisis métrico es el relativo al grosor. Desde esta perspectiva, los anzuelos de La Algaida han sido fabricados con varillas de diferentes grosores, desde aquellas más delgadas $(0,1 \mathrm{~cm})$ hasta las más gruesas $(0,3 \mathrm{~cm})$. En este caso, sí parece advertirse cierta relación entre la altura y los grosores de los ganchos. Así pues, los anzuelos muy pequeños han sido fabricados, en la mayoría de los casos, con varillas de entre 0,1 y $0,15 \mathrm{~cm}$ de grosor, mientras que los ejemplares pequeños y medianos presentan grosores de entre 0,2 (n. $\left.{ }^{\text {os }} 4-6\right)$ y $0,3 \mathrm{~cm}\left(\mathrm{n}^{\mathrm{o}} 3\right)$.

En lo que se refiere al peso, hemos podido comprobar que los valores están directamente relacionados con el tamaño de los anzuelos. De este modo, uno de los ejemplares de mayor tamaño $\left(n^{\circ} 3: 4,4 \mathrm{~cm}\right.$ de altura total) presenta el peso más elevado de todos $(3,25 \mathrm{~g})$, mientras que los anzuelos de ínfimo tamaño no suelen superar $1 \mathrm{~g}$ de peso.

Dentro del modelo de análisis que hemos establecido para el estudio de este tipo de artefactos en época antigua (Vargas 2017b), uno de los aspectos que ha resultado de vital interés ha sido el relativo a las varillas metálicas con las cuales se fabricaban estos ingenios, 


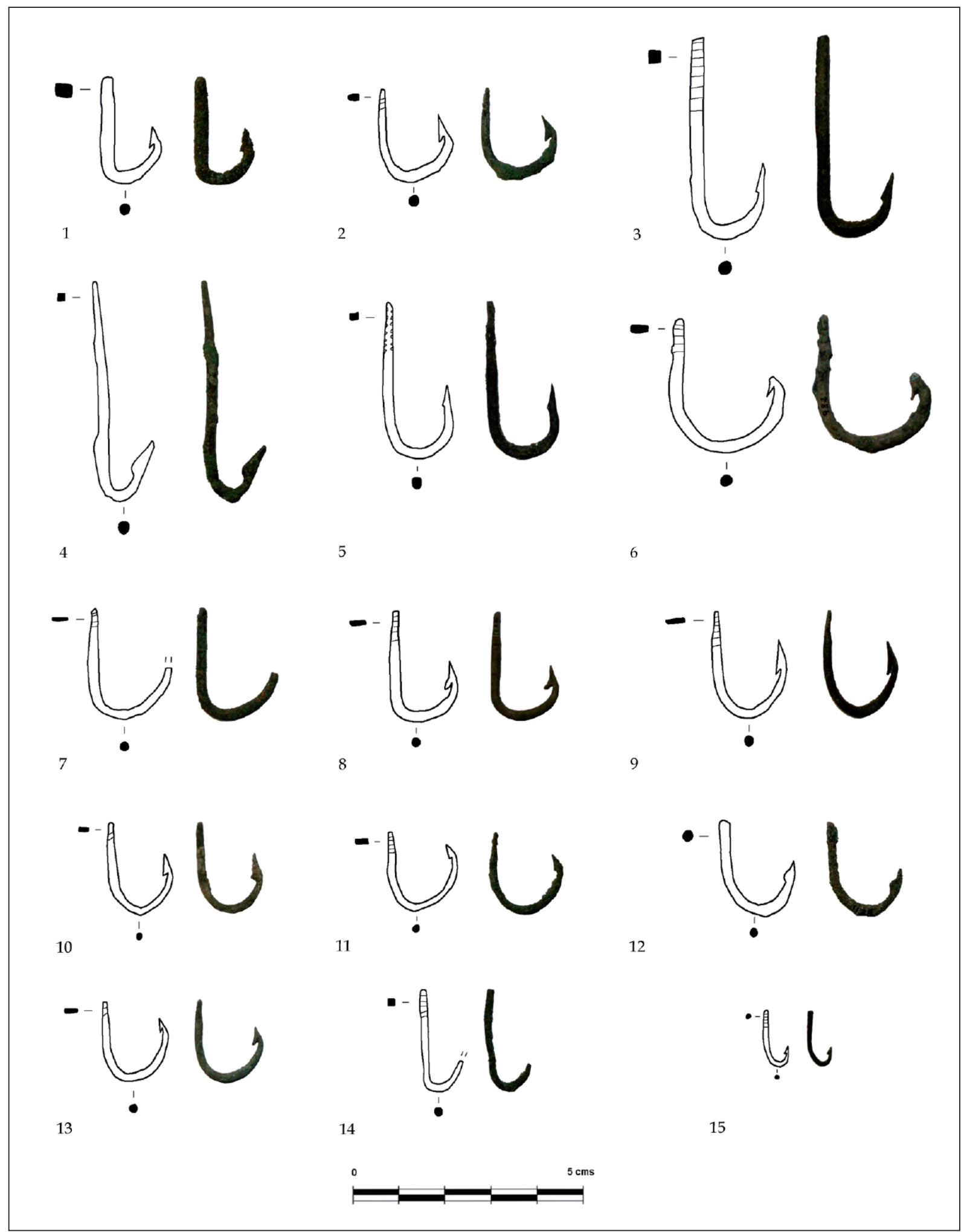

Figura 3. Conjunto de anzuelos simples de La Algaida (a partir de Vargas 2020d). 
OFRENDAS DE CARÁCTER PESQUERO EN SANTUARIOS LITORALES: EL CASO DE LA ALGAIDA...

\begin{tabular}{|c|c|c|c|c|c|c|c|c|c|c|c|c|c|c|c|}
\hline 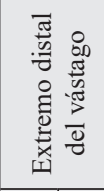 & $\stackrel{0}{.0}$ & 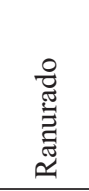 & 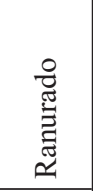 & $\stackrel{0}{\stackrel{n}{3}}$ & 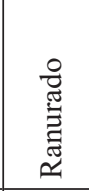 & 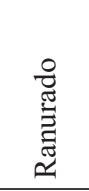 & 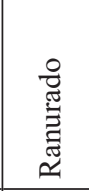 & 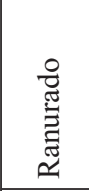 & 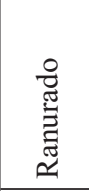 & 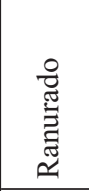 & 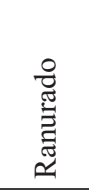 & 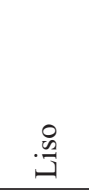 & 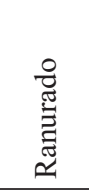 & 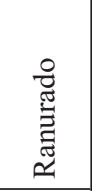 & 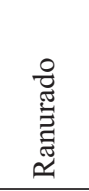 \\
\hline 总 & $\bar{\omega}$ & $\vec{n}$ & $\vec{n}$ & $\vec{\omega}$ & $\vec{n}$ & $\vec{\omega}$ & $\stackrel{\circ}{z}$ & $\vec{\omega}$ & $\vec{\omega}$ & $\vec{\omega}$ & $\vec{n}$ & $\vec{\omega}$ & $\vec{\omega}$ & z & $\vec{\omega}$ \\
\hline \begin{tabular}{l|l}
0 \\
0 \\
0 \\
$\infty$
\end{tabular} & $\bar{\omega}$ & $\bar{\omega}$ & $\vec{n}$ & $\dot{\omega}$ & $\bar{\omega}$ & $\vec{n}$ & $\vec{\omega}$ & $\vec{\omega}$ & $\bar{\omega}$ & $\ddot{\omega}$ & $\ddot{\omega}$ & $\ddot{\omega}$ & $\bar{\omega}$ & $\dot{\omega}$ & $\vec{\omega}$ \\
\hline 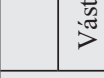 & $\vec{\omega}$ & $\vec{\omega}$ & $\vec{\omega}$ & $\vec{\omega}$ & $\ddot{\omega}$ & $\vec{\omega}$ & $\vec{\omega}$ & $\vec{\omega}$ & $\bar{\omega}$ & $\ddot{\omega}$ & $\vec{\omega}$ & $\ddot{\omega}$ & $\vec{n}$ & $\ddot{\omega}$ & $\ddot{\omega}$ \\
\hline $\begin{array}{l}\tilde{U} \\
\tilde{\Xi} \\
\bar{U} \\
\tilde{y}\end{array}$ & 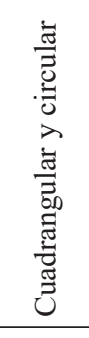 & 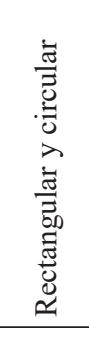 & 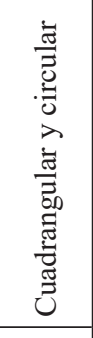 & 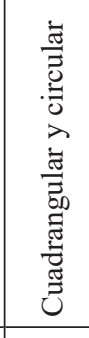 & 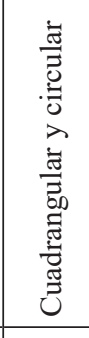 & 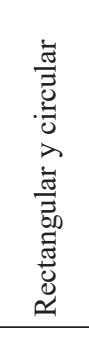 & 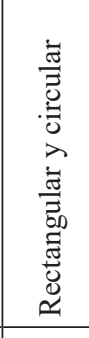 & 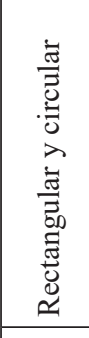 & 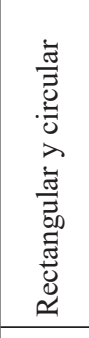 & 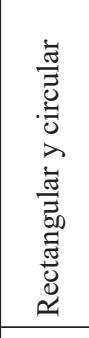 & 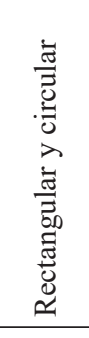 & 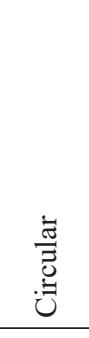 & 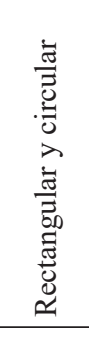 & 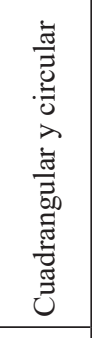 & $\begin{array}{l}\frac{\vec{\pi}}{\bar{E}} \\
\frac{\vec{n}}{0}\end{array}$ \\
\hline 总店 & $\cong$ & $\hat{\sigma}$ & 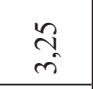 & $\begin{array}{l}n \\
i \\
i\end{array}$ & $\stackrel{8}{-}$ & $\underset{\substack{\infty \\
i}}{i}$ & $\stackrel{n}{\sim}$ & $\underset{-1}{8}$ & $\stackrel{8}{-}$ & 芯 & $\stackrel{\infty}{+}$ & 范 & $\stackrel{0}{\circ}$ & त̂ & $=$ \\
\hline 坖它 & $\begin{array}{c}0 \\
\text { m. } \\
0\end{array}$ & $\stackrel{n}{0}$ & 今. & กิ & กิ & $\begin{array}{l}\text { ते } \\
\text { ô }\end{array}$ & ते & $\frac{n}{0}$ & $\begin{array}{l}\text { กิ } \\
\text { ô }\end{array}$ & $\frac{n}{0}$ & $\stackrel{0}{0}$ & $\frac{n}{0}$ & $\frac{0}{0}$ & $\frac{0}{0}$ & $\frac{0}{0}$ \\
\hline 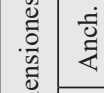 & $\stackrel{\Xi}{\leftrightarrows}$ & $\cong$ & $\cong$ & $\stackrel{+}{\sim}$ & $\stackrel{6}{-}$ & $\begin{array}{l}n \\
i\end{array}$ & $\stackrel{\infty}{-}$ & $\stackrel{n}{\rightarrow}$ & $\cong$ & $\stackrel{+}{\leftrightarrows}$ & $\stackrel{0}{=}$ & $\stackrel{0}{-}$ & $\cong$ & $\stackrel{\circ}{\rightarrow}$ & $\begin{array}{l}0 \\
0\end{array}$ \\
\hline \pm & $\vec{i}$ & $\stackrel{i}{i}$ & $\stackrel{+}{+}$ & $\stackrel{\infty}{+}$ & $\stackrel{+}{m}$ & ते & $\stackrel{+}{i}$ & $\stackrel{+}{i}$ & $\stackrel{m}{i}$ & $\stackrel{i}{i}$ & $\approx$ & $\vec{i}$ & $\approx$ & iv & $\stackrel{\sim}{-}$ \\
\hline 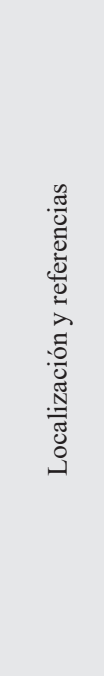 & 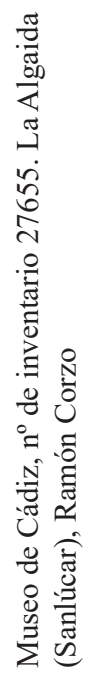 & 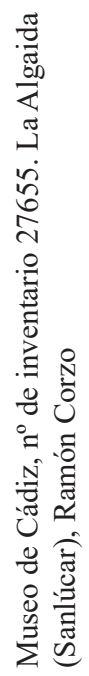 & 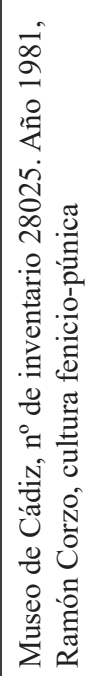 & 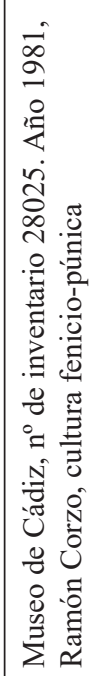 & 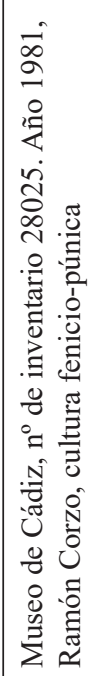 & 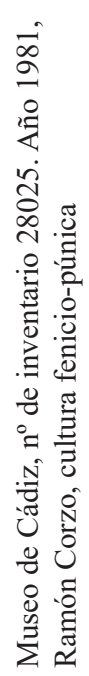 & 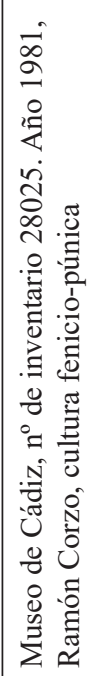 & 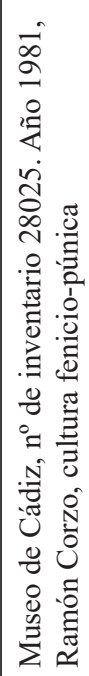 & 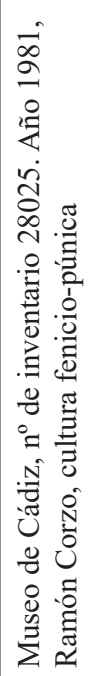 & 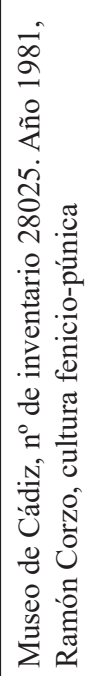 & 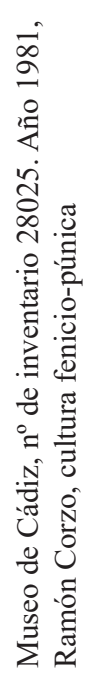 & 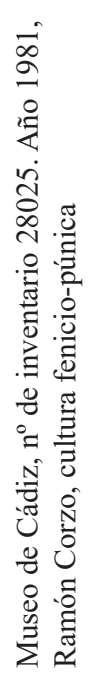 & 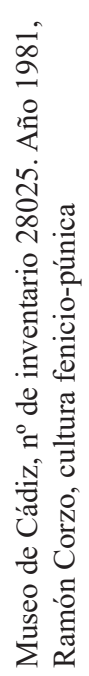 & 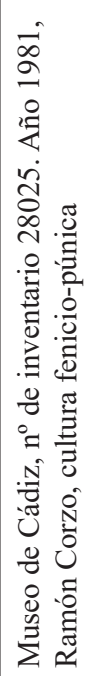 & 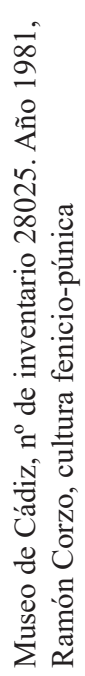 \\
\hline 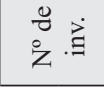 & - & N & $m$ & $\checkmark$ & in & 0 & $r$ & $\infty$ & $a$ & $\stackrel{\varrho}{=}$ & $=$ & $\simeq$ & $\underline{n}$ & \pm & -2 \\
\hline
\end{tabular}


para lo cual ha sido fundamental conocer las secciones de las piezas. Los ganchos de La Algaida se caracterizan por presentar dos tipos de secciones. En primer lugar, contamos mayoritariamente con anzuelos cuya sección es combinada, que son aquellos que presentan diferentes tipos de secciones en la misma pieza. En segundo lugar, aparecen piezas con sección uniforme, que son aquellas que presentan la misma sección a lo largo de toda la pieza. Dentro del primer grupo, hemos identificado dos tipos: secciones circulares y rectangulares $\left(\mathrm{n} .^{\text {os }} 2,6-11,13\right)$ por un lado, y secciones circulares y cuadrangulares (n. ${ }^{\text {os }} 1,3-5$ y 14$)$ por otro lado. Las secciones circulares se observan, en todos los casos, en la mitad inferior del vástago y en la curvatura, mientras que las secciones angulares (cuadrangulares y rectangulares) aparecen en la parte alta del vástago. Esto nos ha permitido esclarecer que la norma general pudo haber sido la utilización de varillas cilíndricas a las cuales se les dio un tratamiento posterior a su fundición -y antes de su total enfriamiento-, sobre todo en el extremo distal del vástago, generándose, de este modo, secciones cuadrangulares y rectangulares. En relación al segundo grupo, aparecen anzuelos con secciones uniformes de tipo circular (n. ${ }^{\text {os }} 12$ y 15 ), que son aquellos ejemplares que no han sufrido ningún tipo de tratamiento posterior. Este tipo de secciones uniformes de tipo circular suelen aplicarse a los anzuelos de ínfimo tamaño cuya fragilidad no soportaría ningún tipo de tratamiento, como puede ser el caso de la pieza $\mathrm{n}^{\circ} 15$, de apenas $1,2 \mathrm{~cm}$ de altura.

En lo que se refiere a los materiales de La Algaida, una de las cuestiones que ha suscitado un mayor interés ha sido el buen estado de conservación en el que han aparecido. En este sentido, las piezas han llegado completas hasta nuestros días, habiéndose conservado en todos los casos -a excepción de los anzuelos n.$^{\text {os }} 7$ y 14- los arpones, que suelen constituir elementos susceptibles de rotura con bastante frecuencia. Por este motivo, se ha podido establecer una clasificación de los ganchos de La Algaida atendiendo a sus arpones, habiéndose elegido dos criterios fundamentales: la forma que presenta su superficie y su orientación. En este sentido, los anzuelos procedentes de este yacimiento pueden presentar arpones bien con superficies rectas (n. ${ }^{\text {os }}$ $1,3,4,10$ y 15) o bien con superficies ligeramente curvas (n. $\left.{ }^{\text {os }} 2,5,6,8,9,11-13\right)$. Por otra parte, los arpones pueden estar orientados de dos maneras posibles: en primer lugar, de forma paralela al vástago (n. ${ }^{\text {os }} 2,6$, 9 y 10) y, en segundo lugar, de manera oblicua con respecto al mismo (n. ${ }^{\text {os }} 1,3-5,8,11-13$ y 15$)$.
Un último aspecto que puede derivarse del estudio de los anzuelos simples es el relacionado con el sistema de sujeción del sedal (Vargas 2017b: 129-130), para lo cual resulta fundamental atender al extremo superior del vástago, que constituye el nexo de unión entre el gancho y la línea. Por lo general, los ejemplares de La Algaida presentan el extremo distal del vástago ranurado (n. $\left.{ }^{\text {os }} 2,3,5-11,13-15\right)$, tal y como viene siendo frecuente en los ejemplares de época fenicio-púnica documentados en la península ibérica (Vargas 2020a). Antes de que se generaran estas ranuras, los anzuelos han sufrido un tratamiento consistente en martillear la parte alta de sus vástagos con el objetivo de generar superficies planas -ya sean de tipo rectangular o cuadrangular- que facilitaran la realización de las incisiones. Por otra parte, hemos documentado tres ejemplares con el extremo distal del vástago liso, que son aquellos que no han sufrido ningún tipo de tratamiento para generar el sistema de sujeción del sedal (n. $.^{\text {os }} 1,4$ y 12). Sin embargo, la presencia de concreciones adheridas en los vástagos de dos de estas piezas (n. ${ }^{\text {os }} 1$ y 12 ) es posible que hayan disimulado las ranuras horizontales para la fijación de la línea.

\subsection{Pesas}

Del santuario de La Algaida proceden un total de catorce pesas de plomo (fig. 4) las cuales responden en todos los casos a modelos laminares, que han sido recientemente tipificados atendiendo, entre otros aspectos, a su forma, habiéndose establecido dos tipos: cuadrangulares y rectangulares (Bernal 2010: 86, fig. 1, PLIX). De las catorce piezas inventariadas tan solo una corresponde al modelo cuadrangular $\left(\mathrm{n}^{\circ} 12\right)$, tratándose de un lastre de dimensiones bastante reducidas $(1,7 \mathrm{x}$ $1,7 \mathrm{~cm})$, de lo que se deduce su escaso peso (18,96 g). La pesa ha sido fabricada a través de una lámina de plomo de apenas $0,9 \mathrm{~cm}$ de grosor que ha sido plegada en dos, generándose un espacio interior de entre $0,1 \mathrm{y}$ $0,3 \mathrm{~cm}$ de anchura, por lo que se aprecia una característica sección en "U" muy frecuente en este tipo de lastres. Esta pieza, junto a otra pesa laminar cuadrangular documentada en la factoría de salazones de Puerto 19 (Puerto de Santa María, Cádiz) y fechada entre mediados del siglo IV y finales del siglo III a.C. (Gutiérrez y Giles 2004: 138 y 139), constituyen los dos únicos ejemplares conocidos, hasta el momento, en el Fretum Gaditanum (Vargas 2020b). En otros entornos del Mediterráneo, este tipo de pesas debieron ser igualmente muy poco frecuentes, ya que tan solo conocemos un 


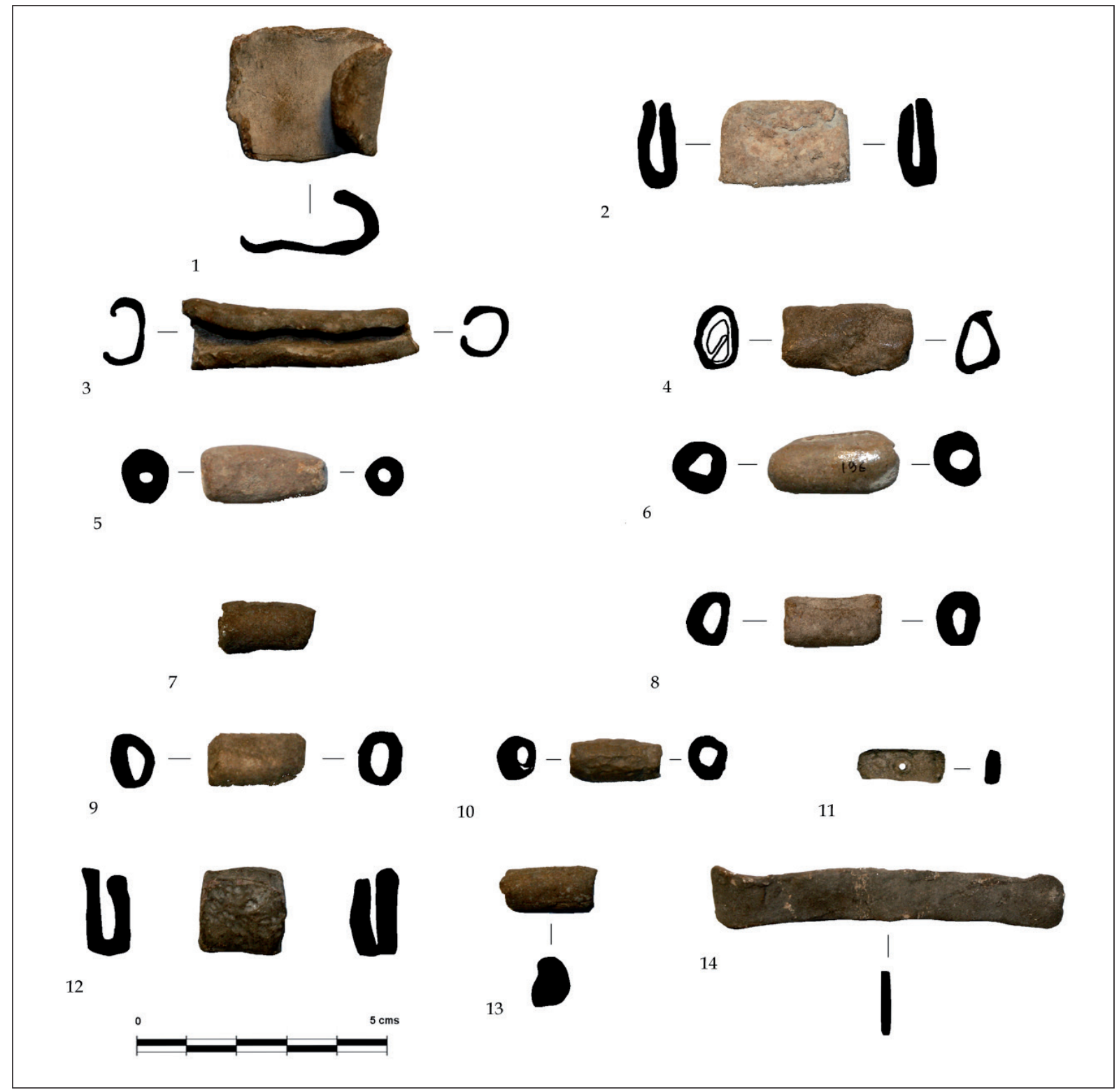

Figura 4. Pesas de plomo de tipo laminar (a partir de Vargas 2020d).

ejemplar procedente del pecio paleobizantino Yassi Ada (siglo VII d.C.), con unas dimensiones no totalmente regulares $-3,6$ × 4,1 x 3,9 x 3,7 cm de lado- $(\mathrm{Ku}-$ niholm 1982: 304, fig. 13-5, LW32).

El resto de las pesas constituyen ejemplares laminares rectangulares, habiéndose inventariado un total de nueve ejemplares que responden a modelos típicamente enrollados (n. $\left.{ }^{\text {ss }} 3-10,13\right)$. Por lo general, son lastres de pequeño formato (entre 1,8 y 2,6 cm de longitud total), registrándose tan solo un ejemplar que supera los $4 \mathrm{~cm}$ de longitud ( $\mathrm{n}^{\circ} 3$ ), y escaso peso. Se observa que la pieza más larga ( $\left.\mathrm{n}^{\circ} 3\right)$ presenta un peso más elevado $(20,29 \mathrm{~g})$ mientras que las pesas con escaso desarrollo longitudinal, como es el caso de la $\mathrm{n}^{\circ} 10$, no llegan a superar los $6 \mathrm{~g}$ de peso $(5,91 \mathrm{~g}$ en este último caso).

Uno de los criterios utilizados para clasificar las pesas laminares rectangulares ha sido el tipo de sección que presentan las piezas (Vargas 2017a), que pueden ser completas o parciales. En el santuario de La Algaida se han documentado ejemplares de los dos tipos. En primer lugar, contamos con un interesante conjunto de pesas con secciones completas (n. $\left.{ }^{\text {os }} 4-6,8-10\right)$, 
que son aquellas de aspecto exterior cilíndrico cuyas secciones aparecen perfectamente cerradas, de lo que se deduce que las láminas estuvieron totalmente enrolladas al cabo de la red. Por lo que respecta a las pesas que presentan una forma totalmente cilíndrica (n. ${ }^{\text {os }} 5$, 6 y 10), se han podido tomar medidas de su diámetro exterior cuyos valores oscilan entre 0,8 y 1,2 cm. Sin embargo, contamos con otros ejemplares que, si bien presentan secciones completas, se caracterizan por presentar un aspecto exterior levemente achatado, de ahí que las medidas que se hayan tomado correspondan a la anchura y al grosor de las piezas. En este sentido, contamos con modelos de lastres más anchos -tal y como es el caso de la pieza $n^{\circ} 4(1,4 \mathrm{~cm})$ - así como otros más estrechos, tal y como demuestran las pesas $n{ }^{\text {os }} 8$ y 9 ( $1 \mathrm{~cm}$ de anchura cada una). En segundo lugar, las excavaciones arqueológicas realizadas en el santuario de La Algaida nos han proporcionado tres pesas laminares rectangulares con secciones parciales o abiertas (n. ${ }^{\text {ss }} 3$, 7 y 13), las cuales podrían haber sido destinadas a lastrar redes cuyos cabos eran de un grosor más elevado que el propio alcance de la pieza, de ahí que no llegaran a abrazar la cuerda completamente, quedando parcialmente abiertas, siendo la pesa $n^{\circ} 3$ el ejemplo más representativo en este sentido. En este caso, la anchura de las piezas está comprendida entre $0,9\left(\mathrm{n}^{\circ} 13\right)$ y $1,4 \mathrm{~cm}$ $\left(n^{\circ} 3\right)$ mientras que los grosores oscilan entre $0,3\left(n^{\circ} 3\right)$ y $0,8 \mathrm{~cm}\left(\mathrm{n}\right.$.s $\left.^{\mathrm{s}} \mathrm{y} 13\right)$.

Una de las cuestiones de mayor interés a la hora de estudiar las pesas laminares enrolladas es el relativo al tamaño del espacio que se ha generado en el interior de las mismas, pues nos informa acerca del grosor del cabo al cual estuvieran adheridas, pudiéndose inferir aspectos tales como la mayor o menor envergadura de las redes. En relación a los ejemplares que presentan secciones completas o cerradas, los diámetros internos de los orificios son muy variables oscilando entre 0,2 y $0,8 \mathrm{~cm}$, aunque las medidas que más se repiten son las de 0,3 (n. ${ }^{\text {ss }} 8$ y 10$)$ y $0,4 \mathrm{~cm}\left(n .^{\text {os }} 6\right.$ y 9$)$.

Si bien la mayoría de las pesas procedentes de La Algaida responden a modelos enrollados, hemos documentado un único ejemplar cuyo sistema de fabricación difiere de los anteriores, tratándose de una lámina doblada o plegada sobre sí misma ( $\left.n^{\circ} 2\right)$, que genera una sección parcial en forma de "U" y un espacio interior mínimo (entre 0,2 y $0,3 \mathrm{~cm}$ ), por lo que suponemos que el cabo de red al cual estuvo adherida era de escaso grosor.

Sin lugar a dudas, una de las piezas que presenta un mayor interés corresponde a la $\mathrm{n}^{0} 1$, pues nos informa acerca de cómo eran originalmente las láminas de plomo a través de las cuales se fabricaban este tipo de lastres en la Antigüedad. Se trata de una lámina en proceso de fabricación, tal y como demuestra uno de sus extremos, parcialmente enrollado. Suponemos que esta pieza no llegó a ser transformada en pesa por enfriamiento del plomo. Se conocen otros ejemplares de láminas en bruto para generar lastres enrollados, como es el caso de una pieza inédita procedente de la factoría púnica gaditana de Plaza Asdrúbal, si bien en este último caso nos encontramos ante un modelo de lámina de mayor formato.

Por último, contamos con un tipo de lastre fabricado a través de una lámina de plomo maciza de grosor variable y de forma rectangular (n. ${ }^{\text {os }} 11$ y 14). Una de ellas responde a un modelo considerablemente largo, alcanzando los $7 \mathrm{~cm}$ de longitud ( $\left.\mathrm{n}^{\mathrm{o}} 14\right)$. Presenta una anchura de $1,2 \mathrm{~cm}$ y un grosor de tan solo $0,2 \mathrm{~cm}$, generándose una pieza muy fina, de ahí su reducido peso (17,50 g). En este caso, no hemos advertido ningún elemento para la sujeción del arte por lo que el cabo de la red debió de ser amarrado directamente sobre la lámina. No parece ocurrir lo mismo con la pesa $n^{0} 11$, constituyendo una pieza de pequeño formato $(1,7 \mathrm{~cm}$ de longitud $\mathrm{x} 0,6 \mathrm{~cm}$ de anchura) y escaso peso $(2,23 \mathrm{~g})$ en cuya zona central presenta un pequeño orificio de $0,2 \mathrm{~cm}$ de diámetro, posiblemente para amarrar el aparejo.

\subsection{Material pesquero complementario}

Incluimos dentro de esta categoría una serie de artefactos, que habitualmente se mezclan con las artes y aparejos de pesca propiamente dichos, que están destinados a fabricar y/o reparar redes y otros enseres de pesca (Vargas 2020c). Nos estamos refiriendo a las agujas y a las lanzaderas.

\subsubsection{Agujas}

Del santuario de La Algaida proceden un total de seis agujas (fig. 5), cinco de las cuales son de bronce (n. ${ }^{\text {s }}$ 1-5), habiéndose documentado tan solo un ejemplar de hueso ( $\left.\mathrm{n}^{\circ} 6\right)$.

Por lo que respecta a las agujas metálicas, aparecen dos modelos bien diferenciados. En primer lugar, contamos con aquellas que presentan un único ojal (n. ${ }^{\text {s }} 1$ y 2). En segundo lugar, aparece un único ejemplar con doble ojal $\left(n^{\circ} 3\right)$. Tan solo una de ellas no ha conservado la cabeza, habiendo sido imposible determinar el número de perforaciones $\left(n^{\circ} 4\right)$. En relación a las agujas 


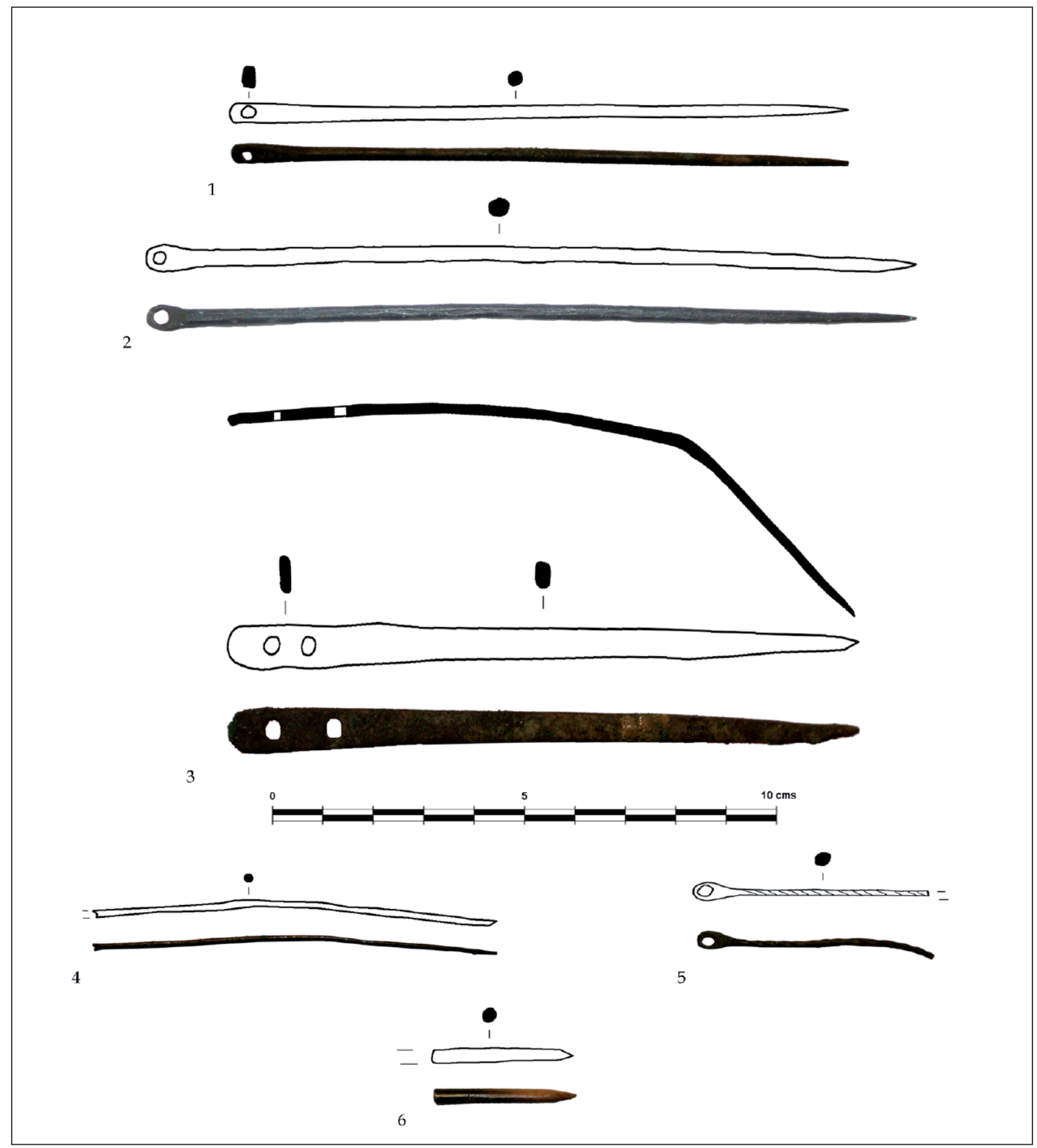

Figura 5. Diferentes modelos de agujas de La Algaida (a partir de Vargas 2020d).

monoperforadas, se caracterizan por estar conformadas por vástagos de sección cilíndrica y cabezas achatadas de sección rectangular, como resultado de haber sufrido un golpe de martillo para generar una superficie plana que facilitaría la perforación del ojal. Los ojales presentan diámetros más o menos regulares, comprendidos entre $0,2\left(n^{\circ} 1\right)$ y $0,3 \mathrm{~cm}\left(\mathrm{n}^{\circ} 2\right)$. En lo que se refiere a sus dimensiones totales, nos encontramos ante un modelo de aguja de gran tamaño, con longitudes de entre $12,4\left(n^{\circ} 1\right)$ y $15,3 \mathrm{~cm}\left(\mathrm{n}^{\mathrm{o}} 2\right)$, de lo que se deduce el grosor de las varillas con las cuales han sido fabricadas ( 0,4 y $0,3 \mathrm{~cm}$ respectivamente). En relación al peso, se 
observa cierta relación con respecto al mayor o menor tamaño de los ejemplares. De este modo, la pieza de mayor longitud $\left(\mathrm{n}^{\circ} 2\right)$ presenta un peso de $9,5 \mathrm{~g}$ frente a los 5,7 g de la pieza $n^{\circ} 1$.

En cuanto a la única aguja con doble ojal que hemos documentado $\left(\mathrm{n}^{\mathrm{o}} 3\right)$, a diferencia de las anteriores, se caracteriza por presentar un vástago de sección rectangular. Constituye igualmente un modelo de gran tamaño (12,5 cm de longitud x $0,7 \mathrm{~cm}$ de anchura) y elevado peso $(9,47 \mathrm{~g})$ con sendos ojales de diámetros desiguales ( $0,3 \mathrm{~cm}$ el ojal superior y $0,2 \mathrm{~cm}$ el ojal inferior).

Por otro lado, contamos con una aguja que no ha conservado su cabeza ( $\left.n^{\circ} 4\right)$, observándose tan solo el arranque de un ojal. Se caracteriza por presentar un vástago cilíndrico de ínfimo grosor $(0,1 \mathrm{~cm})$ por lo que podemos intuir que constituye un modelo monoperforado.

Por último, hemos inventariado una aguja de dudosa atribución $\left(n^{\circ} 5\right)$. Se trata de una pieza caracterizada por presentar un ojal de $0,25 \mathrm{~cm}$ de diámetro y un vástago de sección cilíndrica de $0,1 \mathrm{~cm}$ de grosor en forma de espiral, no habiéndose conservado la punta de la pieza.

Junto a los ejemplares metálicos, hemos inventariado una posible aguja de hueso de la cual solo hemos conservado su extremo apuntado y parte del vástago $\left(n^{\circ} 6\right)$, cuya sección es circular. Al no haber conservado la cabeza, no hemos podido determinar la presencia de ojales, por lo que no podemos afirmar con total seguridad que se trate de un artefacto para coser ya que podría tratarse perfectamente de un punzón o de un elemento de tocado femenino. Un aspecto significativo de esta pieza es la coloración negruzca que ha adquirido, posiblemente por haber estado en contacto con altas temperaturas.

\subsubsection{Lanzaderas}

Del santuario de La Algaida proceden también un conjunto de seis lanzaderas fabricadas en bronce (fig. 6). Las piezas se caracterizan por presentar en todos los casos vástagos de sección circular tal y como viene siendo frecuente en este tipo de útiles, los cuales pueden aparecer rectos (n. ${ }^{\text {os }} 2,3,4$ y 5), ligeramente curvados $\left(n^{\circ} 6\right)$ o notablemente doblados $\left(n^{\circ} 1\right)$. Por su parte, los extremos ahorquillados se han conservado en la mayoría de los casos -a excepción de la pieza $n^{\circ} 5$ que tan solo ha conservado una horquilla, así como en el caso de la $n^{\circ} 4$ que presenta uno de los dos extremos fragmentado-, habiéndose podido constatar que se disponen perpendicularmente uno con respecto a otro. Las horquillas constituyen sendos ojales por donde se bobinaba la fibra. En este sentido, las puntas que conforman los extremos ahorquillados de las lanzaderas nunca pueden llegar a unirse completamente pues, de este modo, impedirían la salida del hilo. Algunas de las lanzaderas que hemos inventariado presentan horquillas cerradas, tal y como demuestran las piezas n ${ }^{\text {os }} 2 \mathrm{y}$ 3 , posiblemente debido a procesos postdeposicionales o a una mala ejecución en el proceso de fabricación, si bien la mayoría de los ejemplares presentan horquillas abiertas, cuyas puntas no llegan a unirse.

En relación a las dimensiones, asistimos a la aparición de un modelo de lanzadera de gran tamaño, con longitudes que superan los $10 \mathrm{~cm}$ e incluso llegando a alcanzar los 16 (n. ${ }^{\text {os }} 3$ y 6) y los $16,3 \mathrm{~cm}$ de $\operatorname{largo}\left(\mathrm{n}^{\mathrm{o}} 2\right)$. En lo que se refiere a los grosores, sabemos que las varillas de fundición utilizadas para la fabricación de estas lanzaderas tenían entre $0,2\left(n{ }^{\circ} 1\right.$ y 5$)$ y $0,3 \mathrm{~cm}$ de grosor (n. ${ }^{\text {s }} 2,4$ y 6$)$.

\section{3. ¿ARTES Y APAREJOS DE PESCA EN SEÑAL DE OFRENDA? LA PRESENCIA DE INSTRUMENTAL PESQUERO EN SANTUARIOS DURANTE LA ANTIGÜEDAD.}

La presencia de instrumentos de pesca en contextos relacionados con santuarios es bastante frecuente durante la Antigüedad. El uso de artilugios pesqueros como exvotos es un fenómeno bien conocido en el mundo griego (Deonna 1938, Gebhard 1998, Mylona 2008, 2015) -fig. 7-aunque sus orígenes podrían remontarse a la Prehistoria Reciente, tal y como han demostrado algunos hallazgos mesopotámicos (Liverani 2014). Es el caso de la cultura de El Ubaid -5100-4000 a.C.- donde el desarrollo de la pesca no solo fue determinante para la dieta de la población, sino que también jugó un importante papel en la esfera religiosa tal y como nos confirman las ofrendas de pescado y utensilios de pesca, los cuales fueron fabricados en las proximidades de los templos (Liverani 2014: 53).

Los primeros testimonios relacionados con evidencias pesqueras en santuarios griegos se tienen constatados desde, al menos, el Geométrico. Así lo han demostrado las excavaciones realizadas en el santuario de Poseidón en Kalaureia -Poros- (fig. 7.1) cuyas investigaciones han revelado actividades de culto y de otra índole desde el Heládico Tardío IIIC -1100 a.C.hasta época romana -siglos I-II d.C.- (Mylona 2015). El instrumental pesquero de este yacimiento está representado por una variedad de pesas de plomo -tanto para 


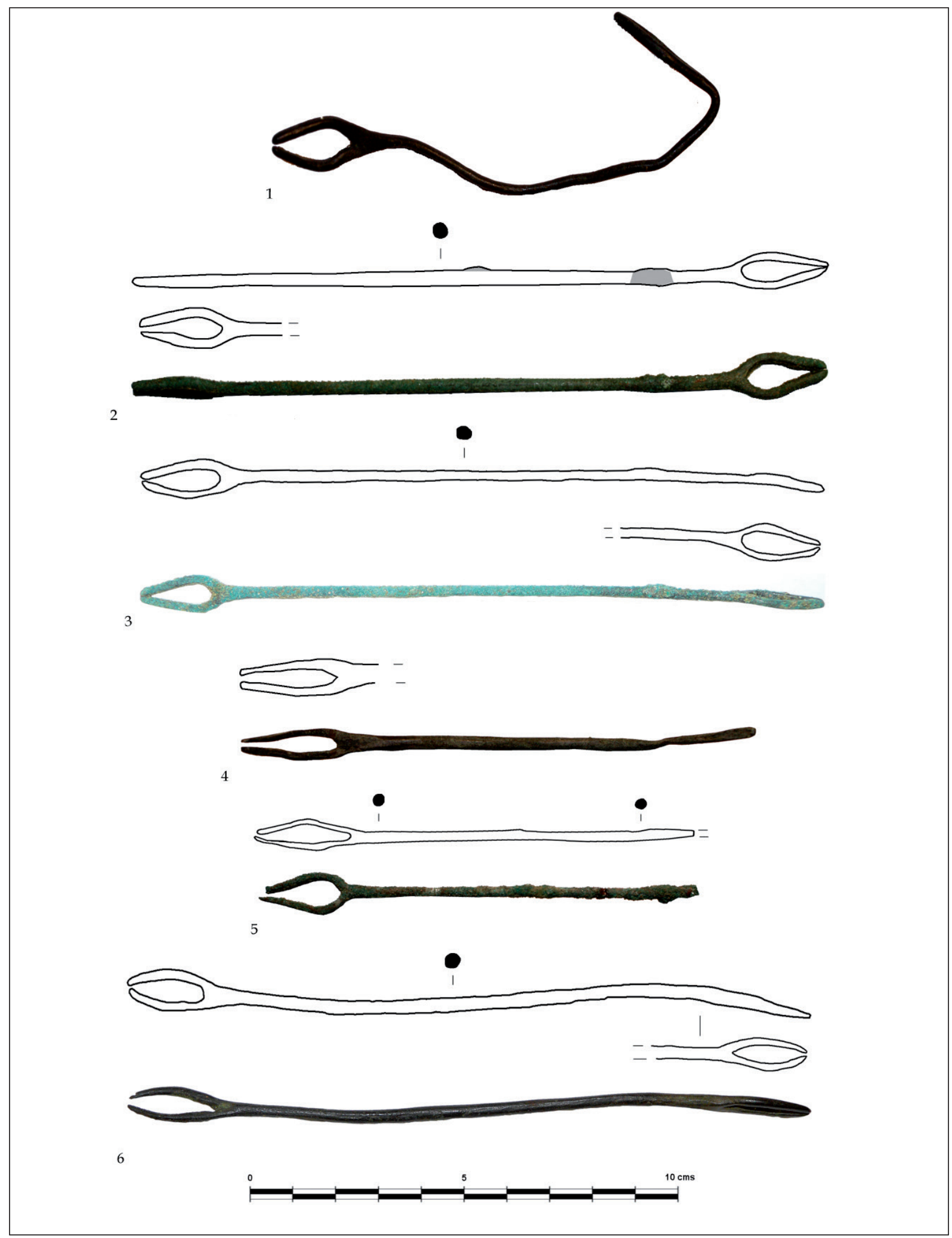

Figura 6. Conjunto de lanzaderas (a partir de Vargas 2020d). 
el lastrado de redes como destinadas a la pesca con caña y/o sedal-que responden a los siguientes tipos: láminas de plomo enrolladas, las conocidas como "lead rings", y pesas cónicas con perforación en el extremo distal, a lo que tenemos que sumar pesas de piedra de posible atribución pesquera y anzuelos de bronce de diversos tamaños (Mylona 2015: 391). También ha aparecido un tridente de bronce que ha sido relacionado con un atributo de la divinidad más que como artefacto de pesca (Mylona 2015: 401).

En relación con el hallazgo de estos materiales de carácter pesquero asistimos a una variedad de contextos, siendo su presencia bastante frecuente en el Geométrico, en época helenística y en época romana altoimperial (Mylona 2015: 391-392). Generalmente, los instrumentos de pesca aparecen en dos zonas:

1) Por un lado, se han encontrado evidencias de instrumental pesquero esparcidas a lo largo del santuario -frecuentemente en niveles de relleno- habiéndose relacionado con ofrendas ofrecidas a la divinidad (Mylona 2015: 391).

2) Por otro lado, mayor información tenemos de los materiales procedentes del denominado "Edificio 1", localizado fuera del recinto del santuario, con varias fases de uso datadas entre época helenística tardía y época romana altoimperial, evolucionando desde una vivienda -fase helenística- hasta una tienda para la venta de comida y pequeños objetos -fase romana(Mylona 2015: 397-399). De la fase romana del edificio proceden numerosos instrumentos de pesca -láminas de plomo rectangulares enrolladas, grandes anillas de bronce, pesa de plomo cónica perforada y anzuelos- habiéndosele asignado dos interpretaciones posibles (Mylona 2015: 399). En primer lugar, es posible que las artes y aparejos de pesca pertenecieran a los pescadores encargados de suministrar productos de origen marino, cuya preparación y servicio, a modo de alimento, tenía lugar en el propio edificio. En segundo lugar, si atendemos al carácter comercial del "Edificio 1" en su fase romana altoimperial, los instrumentos de pesca podrían haber sido objeto de venta a los propios fieles que visitaban el cercano santuario, ya que se han encontrado objetos de similares características esparcidos dentro del mismo. Es posible, incluso, que los objetos de plomo pudieran haberse fabricado en el interior del "Edificio 1" para su venta, ya que se han encontrado evidencias de un uso extensivo de este metal.

No menos interés han tenido las excavaciones realizadas en el santuario arcaico de Poseidón en Isthmia
-Corinto- (fig. 7.2) de cuyos depósitos (A, B, C y D) se ha recuperado un lote de doce pesas de plomo -Depósito A - así como un arpón de pesca fabricado en hierro Depósito C- (Gebhard 1998), todo ello procedente de la pronaos del santuario (Mylona 2008: 137, apéndice 3a). Lo verdaderamente interesante de estos contextos es que en los mismos depósitos donde aparecen los materiales de carácter pesquero, se depositan otras ofrendas tales como aríbalos, jarras fabricadas a mano, monedas, accesorios de carro, joyería y escarabeos (Gebhard 1998).

Entre los años 470-450 a.C. se produce un incendio que destruye el templo arcaico de Poseidón en Isthmia, reconstruyéndose en época clásica (Gebhard 1998). De esta nueva fase del santuario - posiblemente datada a finales del siglo $\mathrm{V}$ o principios del siglo IV a.C.- contamos con el hallazgo de un gran pozo circular relleno con objetos que habrían sido retirados después del incendio que asoló al templo, probablemente durante las labores de limpieza y las tareas de recuperación del mismo. En este pozo apareció una concentración de diecinueve anzuelos de pesca que han sido interpretados como exvotos (Mylona 2008: 137, apéndice 3a).

En la isla de Delos (fig. 7.3) se conocen numerosas localizaciones donde han aparecido artilugios de pesca (Mylona 2008: 137, apéndice 3a). Sabemos que los pescadores de esta isla consagran a los dioses las herramientas utilizadas para ejercer su profesión, tales como tridentes (Deonna 1938: 200), anzuelos de bronce (Deonna 1938: 201), lanzaderas (Deonna 1938: 202) y pesas de plomo piramidales (Deonna 1938: 157). Además de los materiales que acabamos de exponer, se conocen una serie de inscripciones donde se enumeran los exvotos ofrecidos por los fieles a la divinidad, entre los que se encuentran, a menudo, artes de pesca (Mylona 2008: 137, apéndice 3a). Uno de los ejemplos mejor conocidos es el inventario del templo de Zeus Cynthien, donde se cita la consagración de tridentes - uno de los cuales ha perdido su punta mientras que otro ejemplar de hierro apareció completo- y un arpón con dos puntas (Deonna 1938: 200).

Los anzuelos procedentes de esta isla presentan dimensiones muy variadas $\mathrm{y}$, atendiendo al extremo distal de sus vástagos, pueden ser martilleados o ranurados. Resulta de especial interés la aparición de un ejemplar múltiple o potera cuyas piezas (cuatro en total) habrían sido soldadas con un aplique de plomo (Deonna 1938: 201). De igual interés son los anzuelos encadenados aparecidos en esta isla (Deonna 1938: 202) pues actualmente se conocen muy pocos ejemplos procedentes de yacimientos de época antigua a nivel atlántico-mediterráneo 


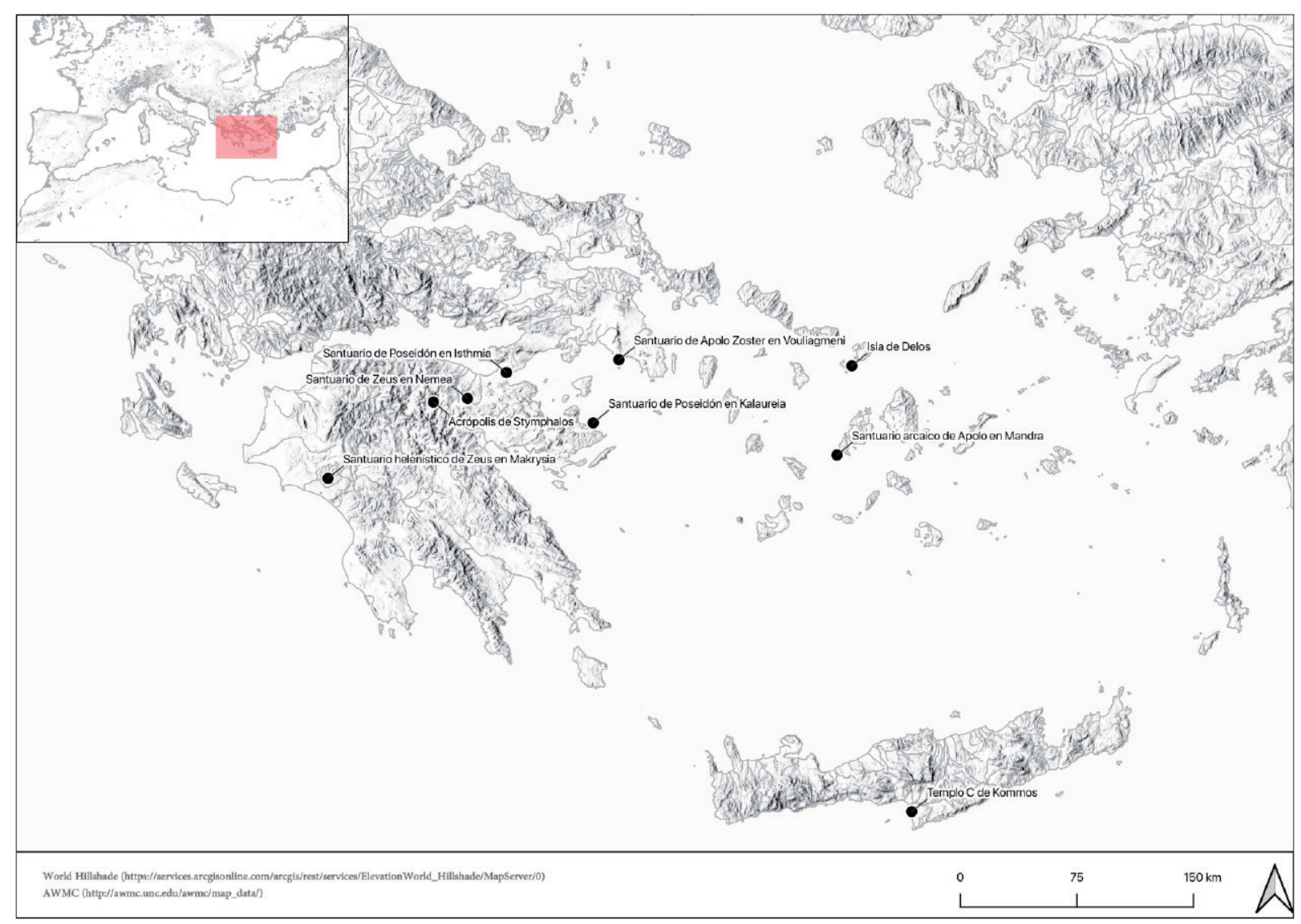

Figura 7. Mapa de dispersión con las localizaciones de santuarios donde ha aparecido instrumental pesquero.

En el Templo C de Kommos (375/350 a.C. - 160/170 d.C.), al sur de Creta (fig. 7.4), se han excavado varios depósitos donde han aparecido una lanzadera de bronce así como dos pesas de plomo para lastrar sedales (Mylona 2008: 138, apéndice 3a).

Del santuario de Zeus en Nemea (fig. 7.5), y más concretamente del sector N16, procede un anzuelo de bronce, si bien el contexto arqueológico donde se produjo el hallazgo de la pieza no permite corroborar que se trate de una ofrenda, ya que este sector se ha visto afectado por actividades agrícolas durante los períodos bizantino y tardorromano (Miller 1976: 183-184). Dimitra Mylona menciona un segundo anzuelo de bronce procedente de la zona periférica del propio santuario (Mylona 2008: 138, apéndice 3a).

En el santuario helenístico de Zeus en Makrysia distrito de Elis- (fig. 7.6), en la orilla del río Alpheios, apareció también un anzuelo de pesca (Mylona 2008: 138, apéndice $3 a$ ).

El área sagrada de la acrópolis de Stymphalos (Corintia), al norte del Peloponeso (fig. 7.7), está conformada por un pequeño templo, un altar y un gran edificio ("Building A") tradicionalmente conocido como "Priest's House" o "casa del sacerdote" (Schaus 2014a: 512). Algunos de los materiales inventariados como "otros objetos utilitarios de bronce" (Schaus 2014b) podrían corresponder a instrumentos de pesca. Es el caso de las agujas - una de las cuales procede del área del altar (Schaus 2014b: 172, $\mathrm{n}^{\circ}$ 133) mientras que otra se ha recuperado en el templo (Schaus 2014b: $\left.172, \mathrm{n}^{\circ} 135\right)-\mathrm{y}$ del único anzuelo de bronce aparecido (Schaus 2014b: 172-173, $\mathrm{n}^{\circ}$ 138), si bien el contexto arqueológico donde se produjo su hallazgo - muralla de la ciudad- no permite corroborar su función votiva. Entre los objetos de plomo contamos con una serie de lastres procedentes del "Edificio A", uno de los cuales -de forma discoidal con hendidura en el borde para sujetar el sedal o el cabo de la red- ha sido interpretado como pesa de pesca (Schaus 2014b: 181, nº 213).

En el sitio arqueológico de Vouliagmeni (Ática), muy cerca de la ciudad de Atenas (fig. 7.8), contamos con el santuario de Apolo Zoster, cuya construcción se 
remonta al menos al siglo VI a.C. (Camp 2001: 316). Muy cerca del santuario se localizó una casa privada en uso durante los siglos V-IV a.C.- cuya pertenencia al sacerdote encargado del culto ha podido determinarse gracias a la aparición de una inscripción y una vasija incisa (Camp 2001: 317). En una de las habitaciones de esta "Priest's House", concretamente en la "Room Z", se documentó un interesante lote de instrumental pesquero conformado por pesas de plomo para redes (seis ejemplares pertenecientes al tipo "lead ring" y veintiséis pesas cilíndricas), cinco anzuelos (tres de los cuales aparecen unidos), cuatro lanzaderas, un cuchillo de obsidiana, una pequeña punta de flecha de bronce y veintidós pesas de telar fabricadas en cerámica interpretadas en este contexto como lastres pesqueros (Mylona 2008: 137, apéndice 3a), aunque tampoco descartamos que pudieran haber servido para tensar las fibras durante la confección de redes, tal y como demuestra la presencia, en este mismo ambiente, de lanzaderas.

Recientes excavaciones en el santuario arcaico de Apolo en Mandra -isla de Despotiko-(fig. 7.9) han permitido descubrir nuevas construcciones pertenecientes a este complejo arquitectónico sagrado, habiéndose documentado objetos de metal entre los que se encuentran un arpón de bronce y anzuelos de pesca ${ }^{1}$.

Por lo que a la península ibérica se refiere, el santuario de La Algaida no ha sido el único espacio sacro donde se han registrado instrumentos de pesca. En este sentido, en el Palacio-Santuario de Cancho Roano (Zalamea de la Serena, Badajoz) se ha documentado un lote de pesas de plomo laminares enrolladas datadas por criterios crono-estratigráficos en el siglo $\mathrm{V}$ a.C. (Mayoral et al. 2000: 192), constituyendo uno de los contextos más antiguos donde han aparecido este tipo de materiales a nivel peninsular. En una de las estancias perimetrales del denominado "Sector Oeste", concretamente en la estancia O-1 (fig. 8.a), se hallaron una serie de lastres de plomo que han sido interpretados como ajuares y ofrendas (fig. 8.b), los cuales fueron depositados intencionalmente en el interior de la citada estancia (Celestino 1996: 33). En relación con los materiales arqueológicos recuperados en $\mathrm{O}-1$, se han distinguido, por un lado, los que se hallaron en la zona norte -carente de pavimento y recogidos en estado fragmentario y disperso- $-y$, por otro lado, los del resto de la habitación, los cuales fueron recuperados completos y colocados

1. Esta información procede de una noticia publicada con fecha 08/08/2018 por parte del Ministerio de Cultura y Deporte: https:// www.archaeology.wiki/blog/2018/08/08/important-finds-in-thesanctuary-of-apollo-on-despotiko/ en el suelo o sobre las banquetas. De la zona norte se recogieron una serie de fragmentos cerámicos sueltos de diferentes tipologías, algunos objetos de bronce, hierro y plomo, entre los que destacamos las pesas laminares enrolladas. Todo ello apareció de manera desordenada entre los núcleos de carbones endurecidos que conformaban el nivel III (Celestino 1996: 36-37). En relación a los lastres plúmbeos, los cuatro ejemplares aparecieron juntos por lo que se ha planteado que pudieron haber estado ligados a un mismo objeto en el momento de su abandono (Celestino 1996: 87). En relación a la función de las estancias documentadas en el Sector Oeste del Palacio-Santuario de Cancho Roano (de O-1 a O-5), se ha planteado que pudo tratarse de capillas de ofrendas, idea que también se ha propuesto para las estancias del tramo norte, adecuándose bastante bien el carácter heterogéneo y multiforme de los depósitos de las estancias occidentales (Celestino 1996: 134).

Sabemos que en el santuario ibicenco de Es Culleram han aparecido siete pesas de red de plomo así como un escandallo. Los materiales, que se encuentran actualmente en proceso de estudio por parte del Museu Arqueològic d'Eivissa i Formentera, fueron publicados en su momento (Almagro y De Fortuny 1971) si bien no fueron valorados como objetos sagrados. Agradecemos a Benjamín Costa, director del citado museo, todos los datos que nos ha proporcionado en relación a este santuario púnico.

\section{CONCLUSIONES}

El estudio del contexto donde se producen los hallazgos de instrumental pesquero procedentes de yacimientos arqueológicos antiguos se está convirtiendo en una novedosa línea de investigación (tab. 2), arrojando un tipo de información muy valiosa para determinar cuáles fueron los diferentes usos y funciones de estos artilugios. Tradicionalmente las investigaciones que se han desarrollado sobre instrumentos y técnicas de pesca de época antigua no se han preocupado por el contexto arqueológico de los materiales (Vargas 2019: 56), desconociéndose los ambientes donde quedaron sepultados. En este sentido, creemos que una de las aportaciones más interesantes de este trabajo ha sido demostrar que el instrumental pesquero no solo jugó un importante papel en la esfera económica, sino que también se ha visto representado en otros contextos como pueden ser santuarios (en calidad de objetos sagrados u ofrendas) así como en otros ambientes -necrópolis (formando parte de los elementos de ajuar)-, rompiendo, por lo tanto, 


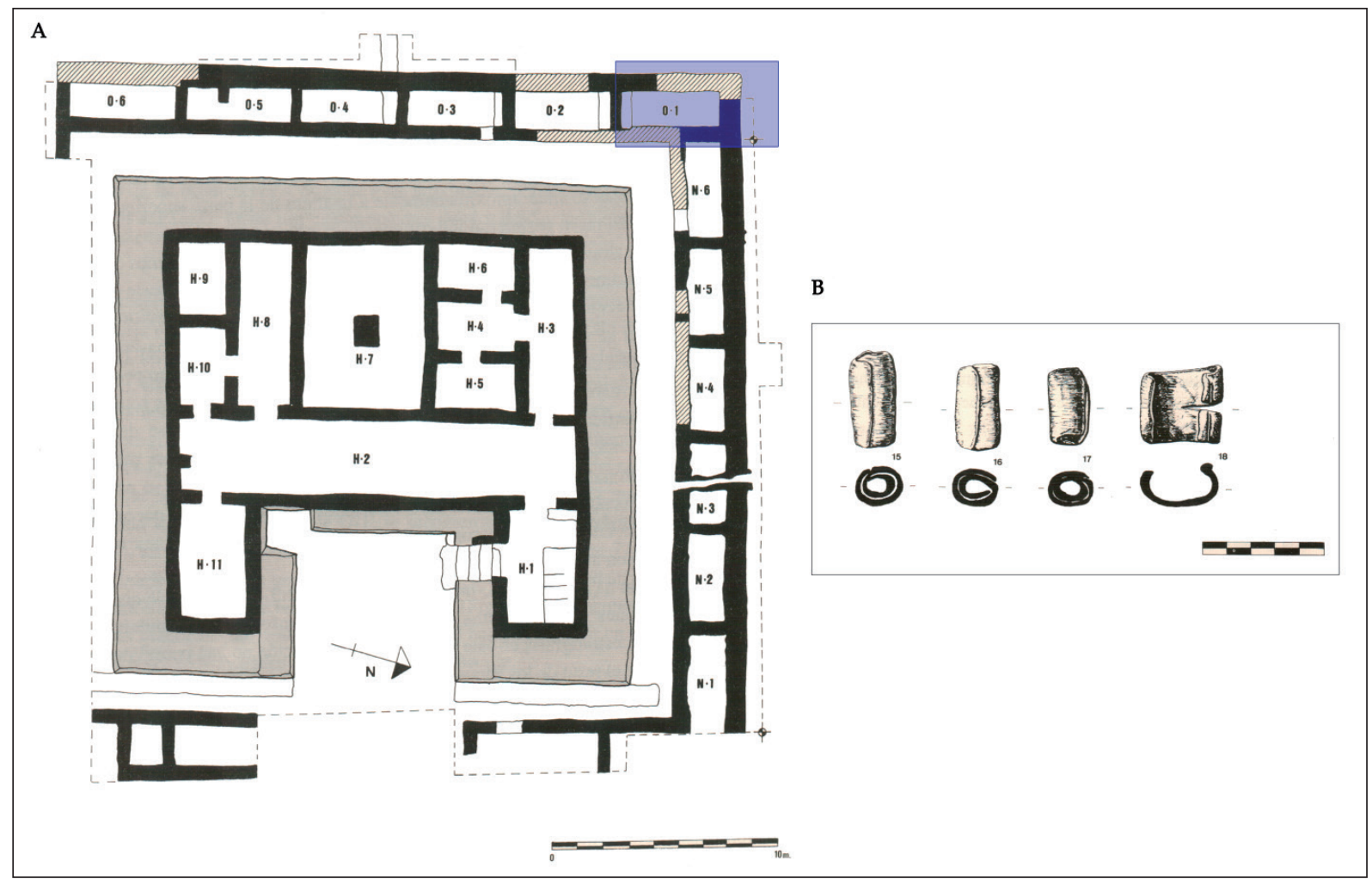

Figura 8. Planta general del complejo monumental de Cancho Roano con la señalización en color azul de la estancia O-1 (A) donde aparecieron una serie de lastres de plomo laminares (B) (a partir de Celestino 1996).

con esa dualidad, tradicionalmente asumida, entre instrumentos de pesca y factoría de salazones.

La presencia de este tipo de material arqueológico en santuarios marítimo-costeros puede explicarse por varias razones. En primer lugar, la razón que parece más evidente es que los instrumentos de pesca se ofrecieran en señal de gratitud y/o veneración a la divinidad o divinidades tutelar/es. En este sentido, los artefactos de pesca formarían parte de los exvotos depositados en el santuario, los cuales podían ser ofrecidos, en primer lugar, por navegantes procedentes de diferentes puntos del Mediterráneo que, tras una larga travesía, entregarían a la deidad los elementos de uso cotidiano durante su vida de a bordo como símbolo de agradecimiento. A este respecto, cabe mencionar que se conocen numerosas localizaciones subacuáticas a lo largo del Mediterráneo que demuestran la frecuente aparición de artilugios de pesca entre los objetos personales de los tripulantes (Parker 1992; Vargas 2014). En segundo lugar, podrían ser los propios pescadores de la zona los que, después de haber obtenido una gran captura, amortizaran sus artes y aparejos de pesca en estos ambientes sagrados en señal de gratitud.
Sea como fuere, la connotación religiosa de estos materiales queda demostrada, en primer lugar, por su aparición en el interior de santuarios. Desde esta perspectiva, los artefactos de pesca podían aparecer dentro del pronaos (siguiendo la terminología griega para designar la estancia delantera de un templo), tal y como hemos tenido ocasión de comprobar en el caso del santuario arcaico de Poseidón en Isthmia (Corinto), así como en el interior de pequeñas capillas destinadas al depósito de ofrendas y ajuares (véase el caso de Cancho Roano). En segundo lugar, son frecuentes los hallazgos de instrumental pesquero en áreas sagradas o témenos, tal y como debió haber sido el caso de La Algaida, a modo de depósitos votivos. Un ejemplo similar lo encontramos en el santuario de Poseidón en Kalaureia (Poros), donde han aparecido elementos de pesca en niveles de relleno esparcidos a lo largo del témenos.

Otro de los ambientes sagrados donde suelen aparecer utensilios pesqueros son las viviendas de los oficiales del culto o sacerdotes, que serían los encargados de custodiar las artes y enseres de pesca, elementos indispensables en estos contextos para 
Tabla 2. Clasificación de los ambientes arqueológicos donde suele aparecer instrumental pesquero en los yacimientos de época antigua.

\begin{tabular}{|l|l|l|}
\hline \multirow{5}{*}{ Yacimientos terrestres } & Contextos industriales & Ambientes pesqueros-conserveros \\
\cline { 2 - 3 } & & Talleres metalúrgicos \\
\cline { 2 - 3 } & Ambientes domésticos & Talleres alfareros \\
\cline { 2 - 3 } Contextos subacuáticos & Ambientes militares \\
\cline { 2 - 3 } & Santuarios marítimos-costeros \\
\hline & Necrópolis \\
\hline & Barcos \\
\cline { 2 - 3 } & Daterial aislado (hallazgos fortuitos) \\
\hline \multirow{5}{*}{ Dragado de puertos } \\
\hline
\end{tabular}

proporcionar los recursos marinos que serían consumidos en los banquetes rituales. En el santuario de La Algaida contamos con interesantes huellas arqueológicas de estas celebraciones tal y como demuestran los numerosos restos de pescado, la propia matriz sedimentaria donde se depositan las ofrendas (arena mezcladas con cenizas y carbones procedentes de actividades culinarias) y la frecuente aparición de platos de pescado. En opinión de algunos investigadores (Marín 2011: 501), la elaboración y celebración de comidas rituales constituyen un paso posterior a los sacrificios, de ahí la abundante aparición de huesos de animales, desconociéndose la existencia de altares para llevarlos a cabo.

Algunos ejemplos de hallazgos de instrumental pesquero en este tipo de viviendas lo encontramos en el "Edificio A" del área sagrada de la acrópolis de Stymphalos (Corintia), así como en la casa privada perteneciente al sacerdote encargado del culto descubierta en el santuario de Apolo Zoster en Vouliagmeni (Ática). En este último caso, además de la función de almacén, la aparición de lanzaderas y pesas de telar de cerámica en una de las habitaciones -"Room Z"-permite plantear el posible carácter artesanal de estas viviendas, pudiendo haberse destinado alguna de las estancias a la fabricación de redes y otros utensilios de pesca. En el caso de La Algaida, se ha planteado que uno de los tres edificios pertenecientes a la fase del santuario podría haber albergado la vivienda de los oficiales del culto si bien no tenemos noticias de la aparición de instrumental pesquero en su interior.
Finalmente, los instrumentos de pesca podían ser vendidos, en calidad de ofrendas, en las inmediaciones de los propios santuarios. Un caso excepcional lo constituye el Edificio I del santuario de Poseidón en Kalaureia que pudo haber servido, en época romana altoimperial, como establecimiento para la compra-venta de comida y pequeños objetos, entre los que se encuentran elementos de pesca. La similitud entre los materiales encontrados esparcidos dentro del santuario y aquellos documentados in situ dentro del Edificio I ha permitido su interpretación como exvotos, pudiendo haber sido fabricados ex professo como tales. Para el caso de La Algaida, se ha planteado la existencia en el propio santuario o en los alrededores, posiblemente en la ciudad de Ébora, de talleres y comercios destinados a la venta de exvotos (Marín 2011: 503).

En relación con la aparición de instrumental pesquero en estos ambientes sagrados, resulta verdaderamente arriesgado valorar si se trata de ofrendas fabricadas ex professo o si por el contrario constituyen simples elementos utilitarios que, finalmente, son amortizados como exvotos. A este respecto, la función de estos materiales podría determinarse conociendo si presentan o no huellas de uso, tal y como se ha planteado recientemente para el caso de las lucernas aparecidas en La Algaida (Marín 2011: 493). A falta de estudios de este tipo, otra de las líneas de trabajo que se presenta como potencialmente más viable para solucionar la problemática función de los materiales aparecidos en contextos sagrados, sería el estado de conservación en el que aparecen las piezas así como su integridad física. 
Para el caso del instrumental pesquero de La Algaida, una de las cuestiones que más nos ha llamado la atención ha sido precisamente el excelente estado de conservación de los anzuelos, pues en la gran mayoría de los casos las piezas aparecen completas, a excepción de los anzuelos n. ${ }^{\text {os }} 7$ y 14 , los cuales no han conservado los arpones. El hecho de que los anzuelos se conserven íntegramente puede ser un indicador interesante para denegar su uso en actividades pesqueras y, por lo tanto, defender su carácter exclusivamente sagrado y su relación con las ofrendas depositadas en el santuario. Otro tipo de instrumental que podría ser analizado desde esta óptica son las lanzaderas. En La Algaida han aparecido dos ejemplares (n.os 2 y 3 ) que presentan, en cada caso, una de sus horquillas totalmente cerrada, imposibilitando la salida de la fibra por este extremo, por lo que su carácter práctico es bastante dudoso. A ello tenemos que sumar que los vástagos de las lanzaderas aparecen totalmente rectos, a diferencia de los ejemplares n. ${ }^{\text {os }} 1$, 4 y 6 que aparecen doblados como resultado de un uso forzado de tales instrumenta. La aparición de agujas y lanzaderas de carácter "utilitario" en este yacimiento resulta de gran interés para valorar la fabricación y/o reparación de artes de redes, trampas (nasas) y otros enseres relacionados con las actividades de pesca en el propio santuario, tal y como hemos tenido ocasión de comprobar en otros contextos del Mediterráneo como es el caso del santuario de Apolo Zoster en Vouliagmeni.

Por todo lo que llevamos dicho, podemos concluir que los instrumentos de pesca procedentes de ambientes sagrados cumplirían dos funciones principales. La primera sería de tipo económico, destinándose el instrumental pesquero a las actividades de pesca llevadas a cabo en el marco de los santuarios marítimo-costeros, en cuyos banquetes rituales cobraba un gran protagonismo el pescado. De la misma manera, si admitimos la permanente residencia de los oficiales del culto en estos enclaves, los recursos marinos constituirían un alimento de fácil accesibilidad en la dieta de los sacerdotes. En segundo lugar, a lo largo de este trabajo se ha pretendido defender otros usos y funciones de los instrumenta piscatoria durante la Antigüedad, los cuales pudieron haber sido depositados en los santuarios de manera intencional como ofrendas, seguramente por oferentes vinculados con el mar entre los que se encontrarían navegantes y pescadores. En este caso, cabría la posibilidad de que fueran exvotos fabricados ex professo para tal fin, pudiendo existir talleres y comercios en el propio entorno de los santuarios que permitieran su fabricación y su venta, sin descartar el "doble" uso de estos materiales, primero como elementos utilitarios -actividades de pesca- y, posteriormente, amortizados como ofrendas -objetos religiosos-.

\section{Agradecimientos}

Este trabajo ha podido realizarse gracias al Proyecto de Investigación de Excelencia Sagena (HUM-03015) financiado por la Consejería de Innovación, Ciencia y Empresa de la Junta de Andalucía. Agradecemos al profesor Darío Bernal Casasola (IP) por habernos cedido los materiales arqueológicos estudiados en el marco de este proyecto para la realización de este artículo.

\section{BIBLIOGRAFÍA}

Almagro Gorbea, M.J. y De Fortuny, E. (1971): "Excavaciones en la Cueva de Es Cuyeram (Ibiza)". Noticiario Arqueológico Hispánico 13-14: 7-35.

Barbadillo, P. (1951): Alrededor de Tartessos. Los descubrimientos de La Algaida, Sanlúcar de Barrameda. Sanlúcar de Barrameda, Santa Teresa.

Bekker-Nielsen, T. y Bernal, D. (2011) (eds.): Ancient nets and fishing gears. Proceedings of the International Workshop on "Nets and fishing gears in Classical Antiquity: a first approach”. Cádiz, Servicio de Publicaciones de la Universidad de Cádiz Aarhus University Press.

Bernal, D. (2009) (ed.): Arqueología de la pesca en el Estrecho de Gibraltar. De la Prehistoria al fin del Mundo Antiguo. Cádiz, Servicio de Publicaciones de la Universidad de Cádiz.

Bernal, D. (2010): "Fishing tackle in Hispania: Reflections, proposals and first results", en T. Bekker-Nielsen y D. Bernal (eds.), Ancient nets and fishing gears. Proceedings of the International Workshop on Nets and fishing gears in Classical Antiquity: a first approach: 83-139. Cádiz, Servicio de Publicaciones de la Universidad de Cádiz - Aarhus University Press.

Bernal, D. (2011) (ed.): Pescar con arte. Fenicios y romanos en el origen de los aparejos andaluces. Catálogo de la exposición. Cádiz, Servicio de Publicaciones de la Universidad de Cádiz.

Blanco Frejeiro, A. y Corzo, R. (1983): "Monte Algaida. Un santuario púnico en la desembocadura del Guadalquivir". Historia 16, 87 (8): 123-128.

Camp, J.M. (2001): The archaeology of Athens. London, Yale University Press.

Celestino, S. (1996): El Palacio Santuario de Cancho Roano V-VI-VII. Los sectores oeste, sur y este. 
Publicaciones del Museo Arqueológico Provincial de Badajoz 3. Madrid, Dirección General del Patrimonio Cultural.

Corzo, R. (1984): "El santuario de la Algaida", en Cádiz y su provincia. Arte Antiguo: 137-171. Sevilla, Gener.

Corzo, R. (1991): "Piezas etruscas del santuario de La Algaida (Sanlúcar de Barrameda)", en J. Remesal y O. Musso (coords.), La presencia de material etrusco en la Península Ibérica: 399-411. Barcelona, Universidad de Barcelona.

Corzo, R. (2000): "El santuario de la Algaida (Sanlúcar de Barrameda, Cádiz) y la formación de sus talleres artesanales", en J.H. Fernández Gómez y B. Costa Ribas (coords.), Santuarios fenicio-púnicos en Iberia y su influencia en los cultos indígenas. XIV Jornadas de Arqueología Fenicio-Púnica: 147-183. Eivissa (1999), Ibiza, Museo Arqueológico de Ibiza y Formentera.

Corzo, R. (2007): "La coroplastia del santuario de La Algaida (Sanlúcar de Barrameda, Cádiz)", en M.C. Marín Ceballos y F. Horn (eds.), Imagen y culto en la Iberia prerromana: los pebeteros en forma de cabeza femenina: 195-218. Sevilla, Secretariado de Publicaciones de la Universidad de Sevilla.

Deonna, W. (1938): Exploration Archéologique de Délos, 18. Les mobilier Délien. Paris, E. de Boccard.

Esteve, M. (1952): "Sanlúcar de Barrameda (Cádiz). Fábrica de salazón romana en la Algaida". Noticiario Arqueológico Hispánico I: 126-133.

Ferrer, E. (2002a): "La religión púnica en Iberia: lugares de culto", en A. González Blanco; G. Matilla Séiquer y A. Egea Vivancos (eds.), El mundo púnico. Religión, antropología y cultura material. Actas del II Congreso Internacional del Mundo Púnico: 107-118. Cartagena (2000), Murcia, Universidad de Murcia.

Ferrer, E. (2002b): "Topografía sagrada del Extremo Occidente: santuarios, templos y lugares de culto de la Iberia púnica", en E. Ferrer (ed.), Ex oriente lux: las religiones orientales antiguas en la Península Ibérica: 185-217. Sevilla, Universidad de Sevilla.

Ferrer, E. (2014): "Ruptura y continuidad en las manifestaciones religiosas púnicas de Iberia (siglos III-I a.C.)", en T. Tortosa (ed.), Diálogo de identidades. Bajo el prisma de las manifestaciones religiosas en el ámbito mediterráneo (s. III a.C.-s. I d.C.). Actas de la reunión científica: 219-250. Mérida (2012), Mérida, CSIC.

Gebhard, R.C. (1998): "Small dedications in the archaic temple of Poseidon at Isthmia", en R. Hägg (ed.), Ancient greek cult practice from the archaeological evidence: Proceedings of the Fourth International Seminar on Ancient Greek Cult: 91-115. Atenas (1993), Stockholm, Åströms Förlag.

Gutiérrez, J.M. y Giles, F. (2004): “36.- Agujas y pesas de red de la factoría de salazones P-19", en A. Arévalo; D. Bernal y A. Torremocha (eds.), Garum y salazones en el Círculo del Estrecho, Catálogo de la Exposición: 138-139. Granada, Ediciones Osuna. Kuniholm, P.I. (1982): “The fishing gear", en G.F. Bass y F. Van Doorninck (eds.), Yassi Ada. A seventhCentury Byzantine Shipwreck, (vol. 1): 296-310. Texas, University Press.

Liverani, M. (2014): The ancient Near East. History, society and economy. London, Routledge.

López Amador, J.J. y Ruiz Gil, J.A. (2010): "Las ofrendas del santuario púnico-gaditano de La Algaida (Sanlúcar de Barrameda)", en E. Mata Almonte (ed.), Cuaternario y Arqueología. Homenaje a Francisco Giles Pacheco: 271-279. Cádiz, Servicio de Publicaciones de la Diputación Provincial de Cádiz - Asociación Profesional del Patrimonio Histórico-Arqueológico de Cádiz.

López de la Orden, M.D. (1990): La Glíptica de la Antigüedad en Andalucía. Cádiz, Servicio de Publicaciones de la Universidad de Cádiz.

López de la Orden, M.D. y Blanco Jiménez, F.J. (2000): "Las monedas de la Algaida (Sanlúcar de Barrameda, Cádiz)", en M. Barthélemy y M.E. Aubet (eds.), Actas del IV Congreso Internacional de Estudios Fenicios y Púnicos, volumen I: 487-508. Cádiz (1995), Cádiz, Servicio de Publicaciones de la Universidad de Cádiz.

Marín Ceballos, M.C. (1987): “Tanit en España”. Lucentum VI: 43-79. https://doi.org/10.14198/LVCENTVM1987.6.04.

Marín Ceballos, M.C. (2011): "Santuarios prerromanos de la costa atlántica andaluza", en M.C. Marín Ceballos (coord.), Cultos y ritos de la Gadir fenicia: 491-532. Cádiz, Servicio de Publicaciones de la Universidad de Cádiz.

Mayoral, V.; Chapa, T.; Pereira, J. y Madrigal, A. (2000): "La pesca fluvial como recurso económico en época ibérica tardía: un ejemplo procedente de los Castellones de Céal (Hinojares, Jaén)". Trabajos de Prehistoria 57 (1): 185-197. https://doi. org/10.3989/tp.2000.v57.i1.269

Meana, M.J. y Piñero, F. (1992): Estrabón. Geografía, Libros III-IV. Madrid, Gredos.

Ménanteau, L. (1982): Les marismas du Guadalquivir. Exemple de transformation d'un paysage alluvial 
au cours du Quaternaire récent. Paris, Université de Paris-Sorbonne.

Miller, G.S. (1976): "Excavations at Nemea, 1975". Hesperia 45: 178-205.

Mylona, D. (2008): Fish-Eating in Greece from the Fifth Century B.C. to the Seventh Century A.D. A story of impoverished fishermen or luxurious fish banquets? BAR International Series 1754. Oxford, BAR Publishing.

Mylona, D. (2015): "From fish bones to fishermen: views from the sanctuary of Poseidon at Kalaureia", en D.C. Haggis y C.M. Antonaccio (eds.), Classical archaeology in context. Theory and practice in excavation in the Greek World: 385-417. Berlin-Boston, Walter de Gruyter.

Muñoz, A. (2012): “Artes y aparejos de pesca feniciopúnicos en el Mediterráneo Occidental: el ejemplo del Círculo del Estrecho", en B. Costa y J.H. Fernández (eds.), Sal, pesca y salazones fenicios en Occidente. XXVI Jornadas de Arqueología Fenicio-Púnica: 33-84. Ibiza (2011), Ibiza, Museo Arqueológico de Ibiza.

Parker, A.J. (1992): Ancient Shipwrecks of the Mediterranean \& the Roman Provinces. BAR International Series 580. Oxford, Tempus Reparatum.

Pérez López, I. (1989): Los santuarios de la Bética en la Antigüedad. Los santuarios de la costa. Tesis doctoral, Universidad de Sevilla. Inédita.

Pérez López, I. (1998): Los santuarios de la Bética en la Antigüedad. Los santuarios de la costa. Cádiz, Servicio de Publicaciones de la Universidad de Cádiz.

Pulak, C. (1988): “A Bronze Age Shipwreck at Ulu Burun, Turkey: 1985 Campaign”. American Journal of Archaeology 92 (1): 1-38. https://doi. org/10.2307/505868.

Ruano, E.; Moreno, R. y Pellus, P. (1996): "Los collares de La Algaida: ofrendas a un santuario gaditano". Boletín de la Asociación Española de Amigos de la Arqueología 36: 107-133.

Schaus, G.P. (2014a): "The temple on the Acropolis of Stymphalos", en D.W. Rupp y J.E. Tomlinson (eds.), Meditations on the diversity of the built environment in the Aegean basin and beyond. Proceedings of a Colloquium in Memory of Frederick E. Winter: 511-529. Stockholm (2012), Atenas, The Canadian Institute in Greece.

Schaus, G.P. (2014b): "Miscellaneous small finds", en G.P. Schaus (ed.), Stymphalos: the Acropolis Sanctuary, (vol. 1): 148-183. Toronto, University of Toronto Press.
Storch de Gracia, J. (1989): La fibula en la Hispania Antigua: las fibulas protohistóricas del suroeste peninsular. Madrid, Universidad Complutense de Madrid.

Vargas, J.M. (2014): "La pesca a bordo en la Antigüedad. Aproximación a través del instrumental pesquero de ARQUA (Cartagena, Murcia)", en X. Nieto y M. Bethencourt (eds.), Arqueología subacuática española. Actas del I Congreso de Arqueología Náutica y Subacuática Española, volumen I: 133-145. Cartagena (2013), Cádiz, Servicio de Publicaciones de la Universidad de Cádiz.

Vargas, J.M. (2017a): El instrumental pesquero en Hispania. Origen, desarrollo y contextualización atlántico-mediterránea. Tesis doctoral, Universidad de Cádiz. Inédita.

Vargas, J.M. (2017b): "El instrumental pesquero en Hispania: estado de la cuestión y perspectivas", en R. González Villaescusa; K. Schörle; F. Gayet y F. Rechin (eds.), L'exploitation des ressources maritimes de l'antiquité. Activités productives et organisation des territoires. XXXVIIe rencontres internationales d'archéologie et d'histoire d'Antibes. XIIe colloque de l'association AGER: 119-137. Antibes, Éditions APDCA.

Vargas, J.M. (2019): "Los estudios sobre instrumental y artes de pesca de la Antigüedad. Balance de la investigación en España y Portugal". Pyrenae 50 (2): 55-83. https://doi.org/10.1344/Pyrenae2019.vol50num2.3.

Vargas, J.M. (2020a): "Los anzuelos de la Antigüedad: modelo de análisis para su investigación e inferencias tecnológicas", en J.M. Vargas (ed.), El instrumental de pesca en el Fretum Gaditanum. Catalogación, análisis tipo-cronológico y comparativa regional: 26-61. Oxford, Archaeopress.

Vargas, J.M. (2020b): "Plomos, piedras y lastres cerámicos: avances en la caracterización tipológica de los contrapesos de pesca”, en J.M. Vargas (ed.), El instrumental de pesca en el Fretum Gaditanum. Catalogación, análisis tipo-cronológico y comparativa regional: 62-117. Oxford, Archaeopress.

Vargas, J.M. (2020c): “Otras evidencias de instrumental y material pesquero complementario", en J.M. Vargas (ed.), El instrumental de pesca en el Fretum Gaditanum. Catalogación, análisis tipo-cronológico y comparativa regional: 118-147. Oxford, Archaeopress.

Vargas, J.M. (2020d) (ed.): El instrumental de pesca en el Fretum Gaditanum. Catalogación, análisis tipocronológico y comparativa regional. Oxford, Archaeopress. 
